
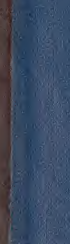

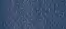
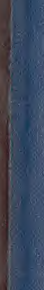

1)

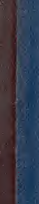




\section{Singaptex:}

\section{C $\triangle \mathrm{T} \perp \mathrm{LOGUE}$.}

of IH:

I'LIVTS LIDER CILTIVATIOS

rx $\tan$

\section{BOTANISAL GARDENS,}

$$
\text { SINGIIPORE, }
$$

\section{STRAITS SETILEYENTS.}
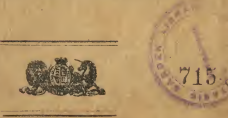

$6 / 4$

$$
\text { SI N G P O R E: }
$$

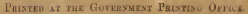

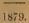




\section{PREFACE.}

Tur Catalogue has been compiled for the purpose of facilitating exchanges of plants, seeds, \&ec, between this and other kindred establishments, as while affording our numerons contributors some data to go on when making out their lists of desiderata, it will serve to show wht plants at present exist in our collection, and thus prevent the loss of time and money which must otherwise inevitably occur.

It is contemplated to publish a Supplement to each Annual Report ma ihe Gardens, containing the names of the plants introduced during the ycar.

A Sumplement to my Annual Report for 1875 contained the names of all the plants then in the Gardens, so far as they were known to me, which amounted to 488 species.

This number in three years has been acarly quadrupled and now amounts to 1,802 .

The families in which the most notable increase has taken place are:- the Orchidex, from 84 to 280 species; Palmis, fiom 44 to 113 ; and Ferns and Lycopods from 31 to 170 species, while only 17 species included in the old list have been lost.

The old list was arrauged alphabetically, which made it easy Nhr reference, and perhaps the number of species in the present one is even row scarcely large enough to warrant my changing the arrangement, but it will render the compilation of future Catalognes mnch easier, and it brings together all the plants that - are nearesi related to each other, and as it is proposed to arrange the slrubby plants themselves in something like the same order, the Catalogue will, in a great measure, answer the porpose of a guide to residents or visitors who are desirons of finding a partieular plant, while an Index of the genera, as well as one containing a good many English and Malay names, have been added to enable non-botanists to find a particular plant. 
The chssification adopted is that of the Genera Plantarum as far as the end of the 2nd Part of the 2nd Volume, after which the orders are given in accordance with the English Bdition of Le Mrout et Decaisne.

With the Aroids, I have followed the alphabetical order as given by Mr. Brown in Sir Joseph Hooker's Report for $187 \%$.

The compilation of even a small Catalogue like the present one in a small Colony like Singapore is attended with a considerable amount of labour and difliculty, and I have been unable to add several particulars for want of the necessary books of reference, \&c.

Although I have taken very great eare to render it as correct as possible, and in revising the proofs, errors have, doubtless, crept in, for which I must beg the indulgenee of the Committer and Public generally.

For the guidance of foreigu contributors, a few general remarks about the climate of Singapore, and the object of this establishment may be useful.

Our Motto may be said to be variety before quantity, consequently, except in the case of useful economic plants, two, or at most three of one species are enough, as the space at our command is not large enough to permit of everything being grown in quantity.

The Island of Singapore produces at the present time but fen plants of beauty or interest, as they have been nearly all exterminated to make way for Nutmegs, Pepper, Thpioea and Gauhbier, \&c., but the adjacent Peninsula is now in a fair way of being opened ont from Johor to Keddah, and a new conntry will thus be open to the Botanist and Gardener, and the mere glimpaes we have had of its vegetation favour the belief that many beautiful and interesting plants exist in the interior of this terra incog. Situated in Lat. $1^{\circ} 17^{*}$ Singapore possesses a fairly equable tropical temperature, and no distinct seasons can be said to exist, as very little, if any, difference exists in the mean average of the temperature of any two months in the year. 
The mean monthly temperature ranges between $78^{\circ}$ and $82^{\circ}$ Fahr. The highest and lowest temperature in the shade during the last ten years being $93^{\circ}$ and $65^{\circ}$ respectively.

The Barometrical readings eorrected and reduced to $32^{\circ}$ Fabr. graduate between $39^{\prime \prime}$ and $30^{\prime \prime}$.

No distinct dry or wot seasons exist, and s week of dry or wet wenther cannot be depended on.

As might be expected, it sometimes rains very heavily, is much as 6.25 inches falling in 24 hourx, and on 27 th Angnst, 18\%8, 5.20 inches fell in about $3 \frac{1}{2}$ hours.

In 1878 , it reached 103.16 inches, while in 1877 it was onfy 58.37 inches, of which 11.47 inches fell in August alone.

The mean annual rainfall for the last 10 years is 91.66 inches.

During these heary rains, anuuals and all tender plants that urc in the open grcund are completely spoiled, such as Zimnias, Balsams, Verbenas, \&c., which during the drier weather serve in no small measure to ornament our grounds.

As no hills of more than 500 fect exist on the Island, it is evident that the task of raising seeds of plants and trees indi. genous in the Temperate Zone cannot be repaid by the result, and shows the uselessness of sending seeds of Norway Spruce, Iarch and Finaster, \&c. here, as some of our well-meaning correspondents have done.

Many plants from the hills of the Peninsula above 5,000 feet refuse to live here and the majority become sickly and fradually die, as for iustance Nepenthes sanguinea nnd Matonia peetinata, which are found in Perak below 4,500 feet.

H. J. MURTON, Superiniendent. 



\section{INDEX OP ENGLISH AND MALAY NAMES.}

The Matax Names iv Itahes.

\begin{tabular}{|c|c|c|c|}
\hline & & & \\
\hline African Oil Palm & 44 & Celery & 16 \\
\hline Alligator Pear & 26 & Cherimoyer & 1 \\
\hline Allspice & 13 & Chiko & 19 \\
\hline American Aloe & 40 & Chickle Gum Tree & 19 \\
\hline Angsane & 10 & Chinese Lantern & 4 \\
\hline Arnatto & 3 & " Tallow Tree & 29 \\
\hline Arrow Root & 31 & Chocolate Tree & 5 \\
\hline Asparagus & 48 & Cinnamon . & 26 \\
\hline Avocado Pear & 26 & $\begin{array}{l}\text { Clove } \\
\text { Cocosinut }\end{array}$ & 14 \\
\hline Balsam of Peru & 10 & Coffee & 17 \\
\hline Bamboo Cane & 50 & "Liberian & 17 \\
\hline Banana & 32 & Cubebs & 30 \\
\hline Bawang-oolin or Kolim & 8 & Cnstard Apple & 2 \\
\hline Bertam & 42 & & \\
\hline Bengal Fig & 27 & Daisy & 18 \\
\hline Betel & 30 & Date Palm & 43 \\
\hline Betel-nut & 41 & Daun Menghok & 16 \\
\hline Blimbing & 6 & Durian & 5 \\
\hline Brake Fern & 51 & Djambo-bidgee & 13 \\
\hline Brazil Rubber & 29 & Dragon's-blood & 48 \\
\hline Bread Pruit Tree or Sookan & 27 & Dukiu & 7 \\
\hline Bullock's Heart & 2 & Divi Divi & 10 \\
\hline Bunga Napatoo & 5 & & \\
\hline Bunya Bunya & 30 & $\begin{array}{l}\text { Earth Nut } \\
\text { Ebony }\end{array}$ & $\begin{array}{l}10 \\
19\end{array}$ \\
\hline Cabbage Palm & 42 & Ebool & 42 \\
\hline Calubash Tree & 23 & Egyptian Paper Plant & 49 \\
\hline Calumander & 19 & Everlasting Pea & 10 \\
\hline Camphor & 26 & & \\
\hline "Malay & 4 & Fennel & 16 \\
\hline Calumba Root & 2 & Fig Tree & 27 \\
\hline Candle Tree & 23 & Flame Tree & 5 \\
\hline Cajuputi & 13 & Flamboyant & 10 \\
\hline Carambola & 6 & Frangipanni & 20 \\
\hline Cardamom & 32 & Funeral Cypress & 30 \\
\hline Cassava & 29 & & \\
\hline Cashew Nut & 9 & Gajam & 10 \\
\hline Ceara-scrap Rubber & 29 & Gamboge & 4 \\
\hline
\end{tabular}


Gamboge, Siam

Ginger

Granadilla

Grape Vine

Gusva

Gum Tree

Gutta jelutong

n) percha

, patih

, sundek

") singgarip

1, taban

\section{Henua}

Herbert Vale Cherry

Holly

Honeysuckle

Ihlang Thlang

Indian Rubber

Indian Shot

Indigo

Ipeeacuanha

Ivy

Jack Fruit

Jamaica Bread Nut

Japan Wax

Jasmine

Jernsalem Artichoke

Job's Tears

Kanarie

Katopany

Kankong

Klappa

Lady's Slipper

Lalany

Lahi

Lanysat

Lemon Gras:

Lemon Tree

Lily

Litehi

Lugrood

Longan

Loquat

Puge.

Mahogany

Page.

32

15

8

13

13

20

19

19

19

19

19

14

29

8

16

$\frac{1}{27}$

31

9

17

16

27

27

9

19

18

50

39

49

5

7

49

49
7

17

8

10

9 Ramel.

12. Rambeh

7

51

9

4

32

25

27

10

30

20

11

47

30

26

26

26

Oil Palm

14

20

Orange Tree

Osage Orange 98

Panama Hat-Palm 44

s. Rubber

27

15

Paraguay Tea 8

Parsley 16

Passion Flower 15

Peepul Tree: - 47

Pepper $\quad 30$

Physic Nut 29

Pinang 41

Pine Apple $\quad 32$

Pitcher Plant 29

Plantain 32

Pomegranate 14

Polasean 9

Prickly Comfrey 21

Pumelow 7

Queensland Nut 26

Quassia 7

27

(9) 


\begin{tabular}{|c|c|c|}
\hline Rambutan & 8 & Tamarind \\
\hline Rhea & & Tea \\
\hline Rice Paper Plant & 16 & Teak or Jati \\
\hline Rakwm & 3 & Tjampedah \\
\hline Manis: & 3 & Temboosie \\
\hline Roselle & 5 & Trabeller's Tree \\
\hline Selak & 43 & Tuba \\
\hline $\begin{array}{l}\text { Salt Bush } \\
\text { Sapodilla }\end{array}$ & 26 & Turmeric \\
\hline $\begin{array}{l}\text { Sapodilla } \\
\text { Sappan }\end{array}$ & 19 & \\
\hline $\begin{array}{l}\text { Sappan } \\
\text { Sarsaparilla }\end{array}$ & 10 & Vmbrella Tree \\
\hline $\begin{array}{l}\text { Sarsaparilla } \\
\text { Satin-wood }\end{array}$ & 47 & \\
\hline $\begin{array}{l}\text { Satin-wood } \\
\text { Sentoel }\end{array}$ & 7 & Vanille \\
\hline Sensitive Plant & 11 & Vegetuble Irory-Sut \\
\hline Sour Sop & 3 & \\
\hline Star Appie & 19 & Watercress \\
\hline St. John's Wort & 3 & Water Lily \\
\hline Sugar Cane & 49 & $\begin{array}{l}\text { Wurinyan } \\
\text { Wild Liquorice }\end{array}$ \\
\hline Tampang & 27 & \\
\hline Tarrap & 27 & Yurrow \\
\hline
\end{tabular}

Page. 



\section{INDEX ORDINUM ET GENERUM.}

Abrus, L.

Abutilon, Gertu.

Acacia, Neck.

Acalypha, L.

Acampe, Lindl.

Acasthacee.

Acanthus.

Achimenes.

Achilles, L.

Achras, L.

Acokanthera, G. Don.

Acrocomin, Mart.

Aerostichnm, L.

Acrotrema, Jack.

Adenanthera, I.

Adinntum, L.

Adinandra, Jack.

Achmea, Ruiz et Par.

Arides, Lour.

Aschvnnuthins, Jack.

Agapanthus, L'Herit.

Agave, 1.

Aglain, Lour.

Aglaonema, Schott.

Albizzia, Duraz.

Aleurites, Forst.

Allamanda, I.

Alocnsin, Schott.

Alphitonia, Reiss.

Alpinia, $\mathrm{L}$.

Alsophíla, R, Br.

Alstonis, R. Br.

Alstromeria, L.

Alternanthera, Forsk.

Alyxis, R. Br.

Anarentacea

A maryllideo

Amaryllis, L.

Amherstis, Wall.

A nomum, L.

Anpelidea
Page.

10
Anacardiacese.

Anacendium, L.

Arcectochilus, $\mathrm{L}$.

Ananassa, Mill.

Andropogou, Willd.

Angelonia, H. et B.

Angiopteris, Hoffm.

Angrecum, Thou.

Anguloa, R, et $\mathrm{P}$.

Anona, L.

Aronaceor

Anthurium, Schott.

Antidesma, Burm.

Antigonum, Bndl.

Antrophyum, Keulf.

Apbelandra, R. Br.

A pium, $\mathbf{L}$.

Apocynacea

Aporum, Blume.

Apostasiacea

Arachis, L.

Aralia, I.

Araliacea

Arsnearia, Henk. \& Hoclust. 80

Ardisia, Sw.

Areca, $\mathrm{L}$.

Arengs, Labill.

Aroideo

Artabotrys, R. Br.

Artanthe, Miq.

Artocerpus, I.

Arum, $\mathbf{L}$.

Arindina, BI.

Arnndo, L.

Asclepiodere

Asclepias, L.

Asperagea

Asnaragopsis, Kth.

Aspurngus, $\mathrm{L}$.

Aspidistra, Gawl

Aspidistrea
Page. 
Axpidium, Swtz. Asplenium, L. Asystasia, Nees. Ataceia, Prest. Attalea, H. ct $\mathbf{K}$. Ancuba, Thumb. Averrhoa, L.

Breckea, I.

Baloghia, Bindl.

Bambusa, Schreb.

Banisteria, L.

Barleria, L.

Barringtonia, Forst.

Basella, L.

Baselleceor

Beaumontia, Wall.

Beeshit, Rheede.

Begonia, L.

Bellis, L.

Bentinckia, Berry

Bignonia, L.

Bignoniacere

Biota, Endl.

Bixa, L,

Bixinece:

Blandfordia, Smith

Blechnum, L,

Boelumerin, Jacq.

Bolbophyllum, Thouars.

Bombax, L.

Boragineor

Bornssus, $\mathrm{L}$.

Bongainvillea, Commers.

Bowenia, F. Muell.

Brainen, IIk.

Brasstia, Endl.

Brassavola, Lindl.

Brussin, R. Br.

Bromeliacem:

Brombeadia, Lind.

Brosimum, Swtz.

Brownea, Jacq.

Brugmansia, Pers,

Brucea, Mill.

Briuasfelsia, L.

Bruguicria, Lam.
Pane.
Bryophyllum, Salieb.

Page.

12

Buckinghamia, F. Muell. Burseracep

Cactem

Casalpinia, Plum, $\quad 10$

Cossalponieor

Culadiam, Vent.

Calamosagus, Grift?

Calarmus, L.

Calnuthe, R. Br.

Calliandra, Benth.

Callicarpa, L.

Callistemon, R, Br.

Caleriendron, Thunb.

Coupanulacere

Cananga, Rumph.

Canariam, Is.

Camucea

Camna, I.

Capprividea

Capparis, L.

Caprifoliacer

Carica, L.

Carissa, $\mathbf{L}$.

Carum, Is.

Caryophyllus, L.

Caryota, I.

Cassia, I.

Castanospermum, A. Cunn. 10

Cattleya, Lindl

Cedrela, I.

Celastrinese

Celtideres

Centradenia, G, Don,

Centropogon Prest.

Cephelis, A. Rich 
Ceratonia, L.

Ceratopteris, Brong

Cerbera, L.

Cereus, Haw.

Chamaerops, L.

Chasalia, Commers.

Chaviea, Mig.

Che nopodeor

Chirita, Humilt.

Chlorenthacea

Chloranthus, Sw.

Chloroxylon, D. C.

Chrysanthemum, I.

Chrysophyllum, I.

Cinnamomum, Burm.

Cirrhopetalum, Lindl.

Cissus, L.

Citrus, L.

Cleisostoma, BI.

Cleome, I.

Clausena, Burm.

Clavija, Ret. P.

Clitoria, L.

Ciivia, L.

Clusia, I.

Coccoloba, Jacq.

Cocos, L.

Calogyne, Lindl.

Coffea, L.

Coix, L.

Colea, Boj.

1 oleus, Lour.

Collabinm, B1.

Colocasia, Schitt.

Colvillea, Bojer

Combretaced

Combretum, L.

Commelyna, $\mathrm{I}$.

Commelyneet

Composite

Conifere

Connaraceos

Connarns, L.

Convolrulacea

Cordyline, Comm.

Comaces

Corypha, L.

Page.

11

52

20

16

43

17

30

26

22

30

50

7
Costus

Crassulacede

Crescentia, L.

Crinum, $\mathbf{L}$.

Crossandra, Salish.

Croton, $\mathrm{L}$.

Cruclfere

Cryptomeria, Don.

Cucrubitaces

Cupania

Cupressus, Tournef

Curenligo Gartn.

Curcuma, L.

Cyathea, Sm.

Cyeas, $\mathrm{L}$.

Cyclanthere

Cymbidinm, Swtz.

Cynometra, L.

Cyperaceat

Cyperus

Cypripedium, L.

Cyrtanthera, Nees.

Cyrtoceras

Cyrtopera, Lindl.

Dacrydium, Soland.

Dalechampia Plum

Dammara, Rumph.

Davallia, Sm.

Delima, $\mathrm{I}$.

Dendrobium, Sw.

Derris, Lour.

Dianella, Lam.

Paye.

32

12

23

41

23

28

2

30

15

8

Dieffenbachia, Schott.

Dillenis, L.

Dillenincert

Dioseorea, L.

Dioscorect.

Diospyros, L.

Diplospora, D. C.

Dipterbearpecee

Dischidia, R. Br.

Dissillaria, F. Mfuell.

Dombeya, Cav.

Doodia, R. Br. 
Doona, Thw.

Doryanthes, R. Br.

Drreana, L.

Drimyspermum, Reinw.

Durantit, L.

Durio, I.

Dryobalanops, Grtn. f.

Drymoglossum, Presl.

Dypsis, Noronh

\section{Elenaceot}

Blreis, Jacq.

Elieocarpus, L.

Elacodendron, Jacq.

Elettarit, Rheode

Elephantopus, Cass.

Fpidendrum, L.

Epiphyllum, Pfeiff.

Branthemum, L.

Bria, L.

Ericacer

Istiobotrya, Lindl.

Brythrine, I.

Erythrospernum, Lam.

Erythroxylon, L.

Eucalyptus, L. Herit

Buchuris, Lindl.

Eugeissonis, Griff

Eurenin, L.

Eulophia, 1.. Br.

Euphorhin, I.

Euphorbicece

Eupomatia, R, Br.

Eurycles, Salsb.

Eutaxia, R. Br.

Eusideroxylon, T. et, B.

Fagrae, Thumb.

Batsin

Faradava, E. Muell.

Fiens, L,

Filices

Pilicium, Thw.

Fittonia, E. Coem.

Flacourtia, Comm.

Flindersia, R. Br.

Foniculum, Adans.

Page.

Fourcroya, Vent.

40 Beanciscea, Pohl.

48 Froycinetia, Gaud.

26 Freycinetice

24 Funkin, Speeng.

5 Garcinit, L.

4 Gurdenia, I.

53

42

Geraniaced

Gesnera, Mart.

Gsneracter

19

41

5

8

32

18

36

16

23

35

18

12

10

3

6

13

41

43

13

36

29

28

Gleditschia, L.
Girroneira, Gaud.

Gleichenia, Sm.

Globba, L.

Gluta, L.

Gruphalium, I.

Goodyera, Lindl.

Gordonia, Ellis

Grammatophyllum, BL.

Graptophylum, Nees.

Grevillen, R. Br.

Grewia, L.

Guilandina, Juss.

Gustavia, L.

Guttifera!

Gymnogramma, Desv.

Gynandropsis, D. C.

Habenaria, Willd.

Hamatoxylon, $L$.

Hakea, Schrud.

Haloragere

Haloragis, Forst.

Hrmanelidea

Hame ia, Jacq.

Hedera, L.

Hedychium, Keuig

Helianthus, $\mathrm{L}$.

Heliconia, L.

Heliotropium, L.

Helminthostaclyys, Kaulf. 5 !

Hevea, Aubl.

Hibiseus, L.

29

Hiptage, Gaertn.

Holmskioldtia, Retx.

Homalomena, Schott.

Houlettia, Brogn.
Page. 


\begin{tabular}{|c|c|c|c|}
\hline & age. & & Page. \\
\hline Hoya, R. Br. & 21 & Lafoensia, Vandelli. & 14 \\
\hline Humboldtia, Vahl. & 11 & Lungeratromin, $\mathbf{L}$. & 14. \\
\hline Hydnoplyytum, Jack. & 17 & Laguuaria, G. Don & 5 \\
\hline Hydranges, $\mathrm{L}_{\text {. }}$. & 12 & Lahia, Hsskl. & \\
\hline Hymenien, $\mathrm{L}$. & 11 & Lansium, Rumph. & \\
\hline Hymenophyllum, $\mathbf{L}$. & 51 & Lantaba & 26 \\
\hline Hymenosporum, F. M uell. & 3 & Lataxia, Comm. & 55 \\
\hline Hyophorbe, Grert. & 42 & Laportea, Gatudich. & 27 \\
\hline Hyporicineo & 3 & Lathyrus: & 10 \\
\hline Hypericum, I. & 3 & Lasrinea & 20 \\
\hline Hypowideat & 41 & Iawsonia, $\mathrm{L}$. & 14 \\
\hline Hyphane & 55 & Lecythis, L. & 14 \\
\hline Iles, I & & Leea, It. & \\
\hline $\begin{array}{l}\text { Hes, it. } \\
\text { Ilicined }\end{array}$ & $\begin{array}{l}8 \\
8\end{array}$ & $\begin{array}{l}\text { Leypumánosip } \\
\text { Lentibudriaceuce }\end{array}$ & 24 \\
\hline Imantophyllum, Hk. & 40 & Leopardanthus, Bi. & 39 \\
\hline Imbricaria, Juss. & 19 & Licuala, Renph. & 48 \\
\hline Imperatin, Cyrill. & 49 & Ligustrum, L. & 19 \\
\hline Indigofera, $\mathrm{L}$. & 9 & Liliacea & 47 \\
\hline Inge, Willd. & 12 & Lillinm, L. & 47 \\
\hline Inocarpus, Forst. & 10 & Limatodes, Blume & 38 \\
\hline Ipoman, L. & 21 & Lindsaya, Dryand. & 51 \\
\hline Iresize, Willd. & 25 & Linea & bi \\
\hline Trideos & 40 & Livistona, R. Br. & 55 \\
\hline Lonandra, $\mathrm{Hk}$. & 19 & Lodoices, Labill & 55 \\
\hline Irotomn, Lindl. & 18 & Loganiaccie & 21 \\
\hline Ixia, L. & 40 & Lomarin, Willd. & 58 \\
\hline Ixora, L. & 17 & Louicera, $\mathrm{L}$. & 16 \\
\hline & & Lorococous & 42 \\
\hline Jaequemontia, Chois. & 21 & tycaste, Lindl. & 38 \\
\hline Jacksonin, R. Br. & 9 & Lycopodiacru & 54 \\
\hline Jambosa, D. C. & 13 & Lyeopodium, L. & 54 \\
\hline Jateorhizn, Miers. & 2 & Lygodinm, Sw. & Fis \\
\hline Jatropha, L. & 29 & Lythrarien & 14 \\
\hline Jonesin, Bosb. & 11 & & \\
\hline Jnbea, H. et K. & 44 & Macadamia, F. Mnell. & 26 \\
\hline Justicin, 1. & 24 & $\begin{array}{l}\text { Machura, Nutt. } \\
\text { Macrozamia, Miq. }\end{array}$ & $\begin{array}{l}28 \\
31\end{array}$ \\
\hline pferia, L. & 82 & Magnolia, L. & 1 \\
\hline Kalanchoe, Adany & 12 & Magnoliaceer & 1 \\
\hline Kentia. Bh. & 42 & Malpighiaced & 6 \\
\hline Kibessa, D. C. & 14 & Malpighia, L, & (6) \\
\hline Kicksie, Blume & 20 & Malnaces & 4 \\
\hline Kopsia, Blume & 20 & Malvaviseus, Dill. & 4 \\
\hline Kyllingia, Rttb. & 49 & $\begin{array}{l}\text { Manmmea, } \mathrm{L} \text {. } \\
\text { Mangifera, } \mathrm{L} \text {. }\end{array}$ & $\begin{array}{l}4 \\
9\end{array}$ \\
\hline Labiat & 25 & Manettia, Mutis & 17 \\
\hline
\end{tabular}


Manihot, Plum.

Maranta, L.

Marattia, J. Sm.

Martinezia, H. \& B.

Mazus, Lour.

Melaleuca, L.

Melanthaceos

Melastoma, L.

Melostomaced

Melin, L.

Melineer

Meriscium, Schreb.

Menispermaced

Меssus, L.

Methonica, Juss.

Meyenia, Schlechtd.

Michelia, L.

Microstylis, Nutt.

Mikamia, Willd.

Milletin, W. et A.

Miltonia, Lindl.

Mimosa, L.

Mimosex

Mimusops, L.

Mirabilis, L.

Monochoria, Presl.

Monstera, Schott.

Morec

Moringa, Brm.

Moringea

Murraya, L.

Musa, $\mathrm{L}$.

Museaces

Mussienda, I.

Myrmecodin, Jack

Myristica, L.

Myristincere

Myroxylon, Nutt.

Myrsined

Myrlaced

Myrtus, L.

Nasturtium, L.

Yehumbium, Juss,

Nipenthea

Nepenthes, L.

Nephelium, L.
Page.

29

31

54

44

28

13

47

14

14
Nephrodium, Rich.

Page.

52

52

20

47

41

53

19

25

.

Ochnacese

Ochnn, Schreb.

Olarined

Oleatera

Olea, $\mathrm{L}$.

Oleandra, Cav.

Onaqrarica

Oncidium Sw.

Oncosperma, B1.

Oplismenis, Palis.

Ophioglossum, L.

Ophiorhiza, I.

Opuntis, Mill.

Orchidese

Oreodoxa, Willd.

Ormosia, Jack.

Orithogalum, L.

Osmunda, L.

Owenin, F. Muell.

Oxalis, L.

Palieouria, Aubl.

Palmee

Panax, $L$.

Pundlaned

Pandanus, L.

Panicum, L.

Papilionacea

Papyrus, Willd.

Pardanthus, Ker.

Parmentiera, D. C.

Phesitlora, L.

Passiftoreo

Pavettia, L.

Payenia, D. C. 
Pedilanthus, Neck.

Pelargonium, L.

Pellen, Link.

Pennantia, Forst.

Pentas, Benth.

Pentstemon, Mitch.

Peperomia, Ruiz et. P.

Pergularia, L.

Persea, Grtn.

Petalostyles, R. Br.

Petiveria, L.

Petrea, L.

Phajus, Lindl.

Phaleenopsis, BI.

Philodendron, Sehott.

Phennicophorium

Phonis, L.

Pholidota, Lind.

Phormium, L.

Photinis, Iindl.

Phrynium, Willd.

Phytelephas, R. et P.

Phylolaccacede

Pierandia, Roxb.

Pilea, Lindl.

Pimenta, Lindl.

Pinanga, BL.

Piperacene

Piper, I.

Pistia, L.

Pithecolobium, Mart.

Pitlasporea

Pittosporum, Banks

Plantaginacese

Plantrgo, L.

Platycerium, Desv.

Plectocomia, Mart.

Plectogyne, Baker

Plocoglottis, BI.

Plumbago, L.

Podocarpus, L'Herit

Poinciana, $\mathbf{I}$.

Poinecttis, Grah.

Polianthes, L.

Polygalere

Polygonea

Polygonum, L.
Page.

29

6

51

8

17

22

30

21

26

11

25

25

36

37

16

43

48

35

17

12

31

4

30

46

12
Polypodium, L.

Polystachya, Hk.

Pometia, Kurz.

Pontederiacere

Porana, Burm.

Portulacea, I.

Portulacacee

Pothos, L.

Pritchardia

Page.

58

35

9

48

21

Proteinophallus, Hk fil. 46

Protenced 26

Psilotium, Sw.

Psychotria, L,

Pterisanthes, Blume.

Pteris, L.

Ptychosperna, Labiel.

Pultenas, Sm.

Punica, L.

Quassia, L.

Quisqualis, L

Reana

Raphistemma, Wall.

Ravenala, Adams

Reidia

Renanthera, Lour.

Rhagodia, R. Br.

Rhomnece

Rhaphiolepsis, Lindl.

Rhapis, L.

Rhizophoree

Phododendwon, $L$.

Rhodoleia, H. K.

Bhodostoma, Schdw.

Rhopaloblasta, Schf.

Rhns, L.

Rhyncosperma, Lindl.

Richarilia, Knth.

Rivinia, L.

Rondeletia, L.

Rosacere

Rourca, Aubl.

Roupellin, Wall.

Rubiacese

Rnsselia, Jacr.

Ruta Lournef.
54

17 
Rutaceu

Sabal Adans.

Saccharum, L.

Secolabium, Blume.

Sagus, Rumpls.

Salvia, L.

Sanchezia, R. et P.

Saadoricum, Cav.

Sanseveria, Thumb.

Sopindacem

Sapotaced

Sarace, L.

Sareanthms, Lindl.

Sarcocephalus, Afi

Scindapsus, Schutt.

Schixata, J. Sm.

Scilla. I.

Scolopendrium, Sta.

Scorodocarpus, Bece.

Scorophitlartincec

Seaforthia, 1i, Br.

Solaginella, P. de B.

Serissa, Commers

Shores, Roxh.

Simarabee

similnce

Smilax

Solandra, Swarty

Solnnace

Solnumm, L.

Solidago, L.

Spathodea, Beauv.

Sputhoglottis, B1.

Spondias, I.

Stachytarpheta, Vahl.

Stanhopen, Lindl.

Stenocarpus, R. Br.

Stephanotis, Thounes

Sterculadea

Sterculia, L.

Stevensonia

Stifltin, Mikau

Stigmaphyllon, Juss.

Stillingia, Garuln.

Strophanthus, D. C.

Stryehnos, L.
Page.

Strelitzia, Banks

Swietenia, L.

43

49

37

43

25

23

7

47

8

19

11

36

17

46

54

47

52

8

22

41

54

18

Symphytum, L.

Tubermamontaria, $\mathrm{L}$.

Taecaced

Tamitis, Swartz.

Tamarindus, L.

Tarrietia, Blnm.

Tecoma, Juss.

Tectons, L.

Templetonia, 1R. Br.

Terminalia, L.

Ternatremiacee

Thelasis, Blume

Theobroms, L.

Thevetia, L.

Thlariantha, Bunga,

Thrinax, L,

Thrixper fistim, Lour.

Thunbergia, $\mathrm{T}_{2}$, til.

Thymeleae

Tilacese

Tinospora, Miers.

Toxicophlas, Sonder

Trapa, L.

Trachclospermum, Lem.

Tredeseantin, L.

Trichopilia, Lindl.

Trichosanthes, I.

Tristelluteia, Thouars.

Tristania, R. Br.

Trithrimax, Murt.

Tritonin, Ker,

Trulbachis, L.

Turnera, D. C.

Thrnerac a

Tydaea, ilene,

Typhonium, Schott.

Encaria, Schreb.

Irceola, Roxb. 20

Etricularia, L. 22

21 Uroskinnera, Lindl.
17

Page.

32

7

21

20

40

53

11

5

23

24

9

13

4

36

5

20

15

43

s9

23

26

5

5

20

14

20

48

39

15 
Vallota, Herb.

Vanda, R. Br.

Vanilla, Plum,

Verbenaceet

Viburnum, L.

Victoria, Lindley

Viminaria, Sm.

Vinca, L.

Vitis, L.

Vittaria, Sw.

Wahlenbergia, Schrad.

Wallichia, Roxb.

Wiekstroemia, Bndl.

Willughbeia, Roxb.

Woodwardia, Sm.

\begin{tabular}{r|l} 
Poge. & \\
11 & Wormia \\
37 & \\
39 & Xanthophyllum, Rxb. \\
24 & Xanthorrea, Sm. \\
16 & Xerotidea \\
2 & \\
9 & Yucea, L. \\
20 & \\
8 & Zalacea, Reinw. \\
53 & Zanthoxylon, L. \\
18 & Zea, L. \\
42 & Zephyranthes, Herb. \\
42 & Zingiberacee \\
26 & Zingiber, L. \\
19 & Zygopetalum \\
52 &
\end{tabular}

Pege.

3

48

48

47

4)

6

49

41

31

32

36

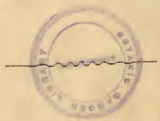




\title{
CATALOGUE OF PLANTS.
}

\author{
EXOGENS.
}

\section{Ond. Dimenniacex, Endl.}

Dehima, $L$.

- sarmentosa, L., Assam to Singapore.

Acrotrema, Jack.

costatum, Hook., Penang and Singapore.

Wormia, Rottb.

pulchella, Jack., Malaces, Singapore.

suffruticosa, Griff $=$ subsessilis, Miry., Mulaya.

Dillenia, $L$.

indiea, $L .=$ exeelsi, Thanb., Ceylon, Singapore.

\section{Ond. Magsoluces, DeCazdolle.}

Magnolia, $L$.

fuscata, Andr, China.

pumila Andr, Amboina.

Michelia, $L$.

Champace, L., E. Indies, Malay Areh. cult.

var. alba.

\section{Oni. Axonacea, Dunal.}

Uvaria, $L$.

Lobbiana, Hook. $f$. et $T$, Singapore.

purpurea, Blene, Singapore.

Artabotrys, R. Br.

suaveolens, Blume, Singapore.

Cananga, Rumph.

Anona, $L$. odorata, Teys., E. Indies, Malaya, Ihlasy Ihlany.

Cherimolia, Mill., W. Indies, Cherimoyer.

Kirkii? Jamaica. 
muricata, Dwr., T'rop. America, Sour Sop.

reticulata, L., S. America, Bullock's Heart.

squamosa, L., Trop. America, Custard Apple.

Eupomatia, R. Br.

laurina, $R, B r$, E. Australia.

\section{Ord. Menispermagen, DeCandolle.}

Jateorhiza, Miers.

palmata, Miers., Mozambique, Culumba root.

Tinospora, Miers.

Fibraurea, Lour.

erispa, Miers., Malaya.

tinctoria, Lowr., Penang, Malacea, Singapore.

Ond. Nyмrнacee, Salisb.

Nymphea, L., Water Lily.

Lotus, $L=$ Devoniensis. Bot. May. 1, 4, 465 . Tropies of old world.

pubescens, Willd, E. Indies.

stellata, Willd., Warmer parts of Asia und Africa. var. cyanea, $H o o k$. $f$, and $T, E$. Indies.

Vietoria, Lindley.

" parviflora, $n$, $n$, $n$

versicolor $y$, ",,$y$,

Regia, Lindley, S. America.

Nelumbium, Jwss.

Leichsrdtii, F. Muell, E. Australia.

speciosnm, Willd., Kashmir to N. Australia.

Ord. Chucifere, Endl.

Nasturtium, $\mathrm{Br}$.

officinale, Br., Temperate Zone, Watercress.

Ord. Capparidex, Vent.

Cleome, $L$.

viscosa, $L$., Tropies-Old and New World.

Gynandropsis, $D, C$.

Capparis, $L$.

pentaphylla, $D . C$.

Mitchellii, Lindl., Austrulia.

versicolor, Griff, Tenasserim, Java. 
Oвр. Morixase, Endl.

\section{Moringa, Brm.}

pterygosperma, Grtn., Malaya.

\section{OrD. Bixixz., Kunth.}

Bixa, $L$.

Orelliaua, $L$, S. America-Arnatto.

Erythrospermum, Lam.

phytolaccoides, Gardner, Ceylon.

Flacourtia, Comm. Roekws.

cataphracta, Roab., India and Malaya. inermis, $R$ Roxb.,

Rukam, Z. et. M., Malaya.

R. Manis.

\section{ORD. Pitrosponge, $R$. Br.}

Pittosporum, Banks

crassifolium, Eanks., New Zealand.

ferrugineum, Ait., E. Australia.

undulatum, Vent., E. Australia.

Hymenosporum, $\boldsymbol{F}$. Muell.

flavum, F. Muell., E. Australia.

Ord, Polyenhaces, Jusgiev.

Xanthophyllum, Raxb.

several unnamed Malayan species.

Ord. Pontulacacra, Jussien.

Portulacea, $L$.

oleracea, $\boldsymbol{L}$., Tropies everywhere.

grandiflora, Camb., Brasil.

Ord. Hrpericinge, DeCandolle.

Hyperieum, L., Sl. John's Worl.

Mysorense, Heyne, Himalayas $3-1000 \mathrm{ft}$.

Orw. Getpifrex, Jussien.

Clusia, $L$.

rosea, $\boldsymbol{L}$, St. Domingo. 
Garcinnia, $L$.

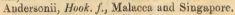

Cambogia, Desr., E. Indies, Ceylon.

Griffithii, T. Andrs., Mount Ophir.

Hanburyii, Hook, f., Siam (True Siam Gamboge.)

Mangostana, L., Malaya. Mangosteen.

Morella, Desr., E. Indies, Ceylon. Ceylon Gamboge.

oblongifolia, Champ., Hongkong.

Xanthoehymus, Hook fil, E. Indies. Burmah.

Calophỵllum, $\boldsymbol{L}$.

inopbyllum $L$, E. Indies to Australia.

Messus, $L$.

Tacamahasa, Willd, Madagascar.

Mamtaea, $L$.,

ferrea, $L .$, E. Indies. Andamans and Ceylon.

amerieans, L., Trop. Ameriea, Mamme Apple.

Orn. Trkxstromacka, DeCandolle.

Adinandra, Jack.

dumosa Jeck, Singapore and Mahn, n.

Gortonia, Ellis.

exeelsa, Blwme, Malaces, \&c.

Camellia, $L$.

var, singapuriana Dyer.

Theifera, Grịj:-, Upper Assam, Cachar, China-Tea.

Ord. Diptennearpacis, Blwme.

Dryobalanops, Grtn. fil.

Shoree, Roxb.

Camphora, Grtn, f. Borneo-Malay Camphor.

Doona, Thw.

stipularis, Thw., Ceylon.

Zeylanica, Thw., Ceylon.

Ond. Manvacen, Jussieu.

Abutilon, Grtn.,-Chizesu Iuniern.

Berangerii, Hort.

Due de Malakoff, Hort.

micropetalum? Australia.

Thompsonii, Hort, fol. variegata.

venosum, H. Teric, Mesico.

vexillarium, E. Morr, Braxil.

Malvaviseus, Dill.

arboreus, Cav., Mexico. 
Hibiscus, $L$.

Cooperii, Hort.

heterophyllus, Vent, E. Australis.

liliflorus? Cav. Mauritius.

Manihot, D.C., China.

moschentos, L., S. America.

mutabilis, $L$, E. Indies.

,. flore pleno.

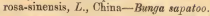

var carneus.

var fulgens.

in flore pleno.

, salmoneus.

tiliaceus, L., E. Indies, Malaya.

Sabdariffa, L., E. Indies, Roselle.

Lagunaria, G. Don.

Patersonii, Don., E, Australia.

Bombax, $L$.

malabaricum, D. C., E. Indies, Malaya.

Durio, $\boldsymbol{L}$.

Zibethinus, 2 G., Malaya-Dwrian.

Lahia, Hsskl,

kntejensis, Hsskl., Borneo-Lahi.

Ord. Sterculacis, Ventenat.

Sterculia, $L$.

acerifolia, A. Cunn, E. Australia-Flame tree.

Bidiwillii Hook, M. Australia.

diversifolia, G. Don. Owaria.

heterophylla, Beaw. Owaria.

laevis, Wall, Penang and Singapore, \&ce.

quadrifide, $\boldsymbol{R}, \boldsymbol{B r}$, $\mathrm{E}$. Anstralis

trichosiphon, Benth., M. Australia.

Tarrietia, Blwme.

argyrodendron, Benth., E. Australia.

Dombeya, Cav.

viburnifolia, Bojer., Manritius.

Theobroma, $\boldsymbol{L}$.

Cacao, L., S. America-Cocoa tree.

Onв. Trlackж, Jussien.

Grewia, $L$.

Microcos, L., Java, Singapore, \&e.

Elueocarpus, $L$.

pexiunculatus, Wall., Malacea \& Singapore. 
Oвр. Linex, DeCandolle.

Erythroxylon, $L$.

Coca, L., Peru-Cacu-leaf tree.

lucidum Moon=Sethia acuminata Thew, Ceylon.

Ord. Malphighraceg, Jusieu.

Malpighis, $L$.

coccifera, L., Trop. America.

Banisteria, $L$.

laurifolia, L., Jamaica.

Stigmaphyllon, A. Juss. ciliatum, A. Juss., Brazil.

Tristellateia, 7 howars.

australiasica, A. Rich., Singapore to Australia.

Hiptage, Gerta.

Madablota, Gertn., E. Indies, Singapore.

Okd. Geraniacka, DeCandolle.

Pelargonium, $L$.

Oxalis, $L$.

quencifolium, Ait, C. G, Hope,

corniculata, L., C. G. Hope,

floribunda, Lehm, Braxil.

A verrhos, $L$.

Bilimbi, L, Native Country unknown Tropics eult. Bilimbing.

Carambola, $\boldsymbol{L}$. do. do. Cardmbola.

ORD. Rutaces, Bartling.

Ruta, $L$.

graveolens, $L$, Europe.

Calodendron, Thusb.

Capense, Thunb., C. G. Hope.

Zanthoxylon, $L$.

nitidum, Roxb. China.

Murraya, $L$.

exotics, L., R. Indies, Singapore.

Clausena, Burm.

Wampi, Blanco, China.

var Pah Toung do.

Citrus, $L$.

Tim do.

aurantium, L., Axia-Orange. 
decumans, L., E. Indies-Pumelovo.

Limetta, Risso, F. Indies-Lime,

Limonium, Risso do. -Lemon.

Medica, L., Asia-Citron.

nobilis, Lour. China, Masularin, Orange.

Ord. Simaruber, DeClandoll.

Quassia, $L$.

amara L. Surinam-Quassia.

Brucea, Mill.

sumatrana, Raxb, Assam to Australia.

Orв. Ochxacex, DeCandollee.

Oehna, Schreb.

atropurparea, D. C, C. G. Hope.

Ord. Burseraces, Kunth.

Canarium. $L$.

commune, L., Malaya, nom, valg. Kanarie.

Filicium, Thw.

decipiens, Thw. Ceylon.

Ord. Mretackx, Adr. Jusieu.

Melia, $\boldsymbol{L}$.

Azedarach, $\boldsymbol{L}$, E. Indies.

Sandoricum, Cav.

indicum, Cav., Rangoon to Singapore-Sentoel.

Aglaia, Lowr. odorata, Lour., China.

Lansium, Rumph.

domesticum, Jack, Malays. Langsat, Dukw.

Owenis, F. Muell.

cerasifera, F. Muell, E. Australin.

Swietenia, $L$.

Mahogani, L., S. America. Mahogasu.

Cedrela, $L$. Toona, Roxb., Trop. Himalayas to Australia, except Ceylon.

Chloroxylon, D. C.

Swietenia, D. C., Concans to Nilghiris, Ceylon. Satis wood.

Flindersia, $\boldsymbol{R}$. $B$ r.

australis, $R . B r$., E. Australia.

Oxleyana, $\boldsymbol{R}$. $B r$., E. Australia, 
Ord. Otacixis, Endl.

Pennautia lorst.

Cunninghamii Evdl. Australia.

Seorodocarpus. Beceari.

borneensis, Becc. Singapore, Borneo Bawang ootan.

Ord. Itichene, Brong.

Ilex, $L$.

aquifolium, L., var. Burope, Holly.

varaguayensis, St. Hill,-Parroguty Tea, Maté.

Okd. Celastranea, $R, B r$.

Elecodendron. Jacq.

australe, Forst, E. Australia.

angustifolium?

Ord. Rhaмxкe, $R$. Brown.

Alphitonia, Reissek.

excelsa, Reissek. Australia.

Ord. AMrguides, Kanth.

Vitis, $L$.

pedata, Vahl., Singapore.

quadrangularis, Wall., India to Java.

$\$$ Cissus. saggitifolis, Laws, Malaya.

vinifera, $L$. Orient., Grape rine.

discolor. Blume Java.

porphryophyllus, Lindley, Singapore.

Pterisanthes, Blume.

Leen, $L$. polita, Miq., Singapore.

sambucina, Willd. India to Australia.

Ord. Saptndackis, Jusieu.

Cupania, $L$.

australis, $\boldsymbol{R} . \boldsymbol{B r}$., B. Australia.

Nephelium, $L$.

lappaceum, L. Malaya, Rambutan.

Lichi, Wght., China. Litchee. 
Lougaua, Bl. China. Longen. mutabile, Bl. Malaya. Polvesan.

Pometiu, Kurz. tomentosa, Kurs. Malacea, Ceylon, sce.

\section{OkD. Axacardiacen, Lindl.}

Rhus, $L$.

succelanea, L. Japan. Japan wax.

Pierardis, Roxb.

dulcis, Jeck Malaya Rambeh.

Mangifera, $L$.

indica, $L$., E. Indies, Mango. 25 named varieties from India.

Anacardium, Rottb. Cashero nut.

Glute, $L$.

oceidentale, $L$., America, elsewhere naturalized.

Benghas, L. Singapore Redivood of Singapore.

Spondiss, $\boldsymbol{L}$. pleiogyna, ? A istralia.

Ord. Connakacze, $L$.

Connarus, $L$.

santaloides, Vahl., Manilla.

Rourea, Aubl.

fulgens, Planch, Singapore.

Ord. Legurtrosis, Jusgieu

Sub. Ord. I. Papilionacian.

Jacksonia, R. Br. scoparia, $R . B r$., N. S. Wales.

Viminaris, $8 \mathrm{~m}$. denudata, Sm., S. Australis.

Pulteniea, Sim. prostrata, $R . B r$, S. Australia,

Entaxie, R. Br. empetrifolia, Schlecht. Australia.

Templetonia, $\boldsymbol{R} \boldsymbol{B}$. retusa, $R . B r$, S. Australia.

Indigofers, $\boldsymbol{L}$. Indigo. australis, Willd. Australia. tinctoria, $L$. India.

Milletia, $W$. et $A$. megasperma ? Australia. 
Arachis, L. Earth wut.

Lathyrus, $L$.

hypogæa, $L$. S. Ameriea.

latifolins, $L$, Furope-Everlasting $P$ ea.

Abrus, $L$.

precatorius, L. E. Indies to Australia-Wild Liquorice.

Clitoria, $L$.

Ternates, L., E. Tndies.

Erythrina, $\mathcal{L}$.

Crista-Gaili, L., Brazil.

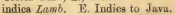

,. fol, aurea. variegata, Hort $_{\mathrm{A}}=$ picta, $L$.

ovalifolia, $R \cdot x b$. Assam to Java.

Parcelli, Bapt., S. S. Islands.

vespertilio, Benth. N. Australia.

Pterocarpus, $\boldsymbol{L}$.

indicus, L., E. Indies, Malaya, China, Angsana.

Derris, Loar.

elliptica? Malaya--Tuba.

Inocarpus, Forst.

edulis, $I$. Malaya-Gajam.

Castanospermum. A. Cunn.

nustrale, A. Cunn., E. Australia. Moreton Bay chesmut.

Ormosia, Jack.

emar finata, Benth., Hongkong.

Myroxylon, $L$. fil.

peruiferum, L. fil. Peru, Balsam of Peru.

Sub. Ord. II. Casalpixga.

Cresalpinia, Plum.

Coriaria, $W i l l d$, S. America-Divi Divi.

Sappan, L., B. Indies-Sappan.

Guilnndine, Juss..

Bonduc, $L$, E. Indies, Malaya.

Poinciona, $L$.

Bonducella, $L$., E. Indies, Malaya.

pulcherrima, L., E. Indies.

var, flore flava.

regia, Bojer Madagascar-Flamboyant.

var, - varicgata.

Hamatoxylon, $I$

Campechianum, L., S. America-Lognood.

Gleditschis, $L$.

sinensis, Lam, China.

Colvillea, Bojer.

Cassis, $\boldsymbol{L}$.

racemosa, Boj, Madagascar.

alata, L., Malaya. 
bacillaris, $\boldsymbol{L}$., Surinam.

Fistula, L, Egypt.

florids, Vahl., Florida.

suffruticosa, $K \in n, \mathbf{E}$. Indies.

sumatrana?

Petalostylis, $R$. $B r$.

labicheoides, $\boldsymbol{R} . B r$, S. Australia.

Ceratonia, L. Carob tree.

Bauhinia, $L$. Siliqua, $\boldsymbol{L}$, Levant.

purpurea, $L$, B. Indies.

scandens, $L_{,}$E. Indies.

tomentosi, $L_{\text {, }}$ Ceylon.

variegata, $L_{i}, \mathbf{E}$. Indies.

Brownea, Jacq.

coccinen Jacq., 8. Ameries.

Amherstis, Wall.

nobilis, Wall., Martarbau.

Humboldtia, Vahl.

laurifolia, Vahl. Ceylon.

Tamarindus, $L$.

indien $L$; E. Indies. Trmarind.

Hymenrea, $I$.

vermacosa, Garts. Madagascar.

Saraca, $L=$ Jonesia, Roxb.

deelinata, Miq., Java.

indica, Miq., E. Indies=J. Asoca, Roxb.

Csnometra, $L$.

esuliflora, L. E. Indies Nom-nam.

Sur. Ond, Mrmosez.

Adenanthera, $\boldsymbol{L}$.

Pavonina, $\boldsymbol{L}$, E. Indies, \& c.

Mimosa, $L$.

Acacia, Neck.

pudica $L_{v}$, 8. America, Sensitive plant.

decurrens, Willd, Australia.

var. mollisima.

Farmesiana, Willd., B. Indies, Australia.

horrida, Willd. Africa.

leprusa, Sieb., Autralis.

linearis, Sims., Australia.

lunata, Sieb., Australis.

salicina, Lindl., Australia.

saligna, Wendl., W. Australia.

spinescens, Benth., Australia. 
Calliandra, Benth. brevipes, Benth., S. America. hematocephala, Hasskl., S. America.

Albizzia, Duras.

lophanths, Benth., W. Australia.

Pithecolobium, Mart.

Inga, Willd.

pruiposum, Benth., E. Australia.

Saman, Willd., Caracas.

Ors. Rosacen, Jussien.

Rhaphiolepis, Lindl. indiea, $L_{\text {indl., China. }}$

Photinia, Benth. \& Hook, fil.=Eriobotrya, Lindl. japoniea, Iandl. China, Loquat.

Ord. Saxiprafacez, Vent.

Hydrangen, $L$.

japonica, Sieb., Japan.

Ord. Crusselaces, DeCandolle.

Bryophyllum, Salisb.

calycinum, salisb., Singapore.

Kalanchoe, Adans.

laciniata, D. C., E. Indies.

Echeverie, D.C.

atropurpurea, Hort.

Ord. Hamametidex, $\mathrm{Br}$.

Hhodoleia, Hook.

Championii, Ht., Hongkong.

Ore. Halorugro, $\mathrm{Br}$.

Haloragis, Forst.

distichs, Baill, Singspore.

Oвd. RHzорнову $\&, ~ L$.

Bruguiera, Lam.

cylindrica, Bl, Java and Singapore. 
Ord. Combretaces, $\mathrm{Bi}$.

Terminalia, L. Katapang.

Catappa, $L$., E. and W. Indies.

melenocarpa, Benth., Australia.

Combretum, $L$.

grandiflorum, G. Don., Sierra Leone.

Krauseanum, Hochst., C. G. Hope.

micranthum, G. Don., Seirra Leone.

Quisqualis, $L$.

indica, $L$, E. Indies.

Ord. MYrtaces, $\mathrm{Br}$.

Backea, $L$.

frutesceus, $L$, China.

Callistemon, $R$. $B r$.

brachyandrus, Lindl,, Australia.

speciosus, D. O., B Australin.

Melaleuca, $L$,

leucadendron, $L_{\text {, }}$ W. Australia E. Indies, Cajuputi.

Preissiana, Bchau., Australia.

Eucalyptus, $L_{\text {, }}$ Herit. Gum tree.

amygdalina, Labill, E. Australia.

Baileyii, F. Mfuell., Queensland.

ealophylla, $\boldsymbol{R}$. Bro, W. Australia.

citriodora, Hook., B. Australia.

Globulus, Labill., Tesmania.

goniocalyx, F. Muell., S. Australia.

Gunnii, Hook., E. Australia.

marginata, Sm., W. Anstralia.

meliodora?

obliqua, L. Herit., S. Australia.

pilularis, Sm., E, Australia.

rostrats, Schlecht., Australia.

Tristanis, $R$. Br.

conferta, $R$. Br., E. Australia.

Psidium, $L$, Guava.

acre?

Cattleyanum, Lindl., S. Ameries.

Guava, $L$, S. America. Djambe bidgee.

var. pyriferum.

Guinense Swarts., S. America.

Myrtus, $L$.

australis? Australia.

spectabilis, Blume, Malaya.

Pimenta, Lindl. -Allspice. vulgaris, Lindl., W. Indies.

Eugenia, $L$., incl. Jambosa. aquea, D. C., Malaya. 
domestica, Rumph., Malays. bypoleuca, Thw., Ceylon.

Jambosa, $L$., E. Indies.

Smithii, Poir., E. Australia.

Ventenatii, Benth., S. America.

xanthocarpe, Thw., Ceylon.

3 Jossinis, Lam.

buxifolia, Lam., Mauritius

Caryophyllus, $L_{\text {, }}$-Clove.

aromaticus, $L_{\text {., Molnccas. }}$

Barringtonia, Forst.

speciosa, $L_{*}$, Moluecas.

Gustavia, $L$.

braxiliaua, D. O., Brasil.

Lecythis, $L$.

minor, Jacq.. S. America

Ord. MeL,

Centradenia, G, Don.

rosea, Dow., S. America.

Melastome, $L$

malabathrica, $L_{,}$, F. Indips, Malaya.

Kibesєa, $D, O$,

axurea, Bl, Singapore

Ond, Lxthranirf, Jussien.

Lafoensia, Vandelli.

miorophylla, $P_{0} h l$, Brazil.

Lawsonia, $L$.

inermis, $L_{*}$, Fgypt. Henna.

Lagerstromia, $L$.

floribunda, Jack, E. Indies.

indica, $L_{\text {, }}$, E. Indies. China, Japan.

Regina, Raxb., E. Indies, Malaya.

Punier, $L_{\text {. }}$ Homegranate.

Granitum, $L$, S. Europe

" flore pleno.

Ond. Oxsgraur.s, Benth, and Hook. fid.

Trapa, $L$.

eochinchinensis, Lour:, Chima. 
Orw. Turneracex, DeCandolle.

Turners, $L$.

trioniiflori, $H k$., Brazil.

Ond. Passinlokk. Juss.

Passiflora, $L$., Passion flower. cerrulea, L., Brazil. edulis, Sims., W. Indies.

feetida, Cav., S. America, naturalized in Singapore.

kermesina, Link., Brazil.

holoserica, L., Vera Crus.

laurifolia, L., S. America.

macrocarpa, Hort., Rio Negro.

quadrangularis, L., S. America, Granadilla.

trifasciata, Lem., Brazil.

Carica, $L$., Papaw tree.

Papaya, L., S. America.

Ord. Cucurbitace.s, Jusi.

Trichosanthes, $L$.

laciniosa, Klein., India to Java.

Thladiantha, Bunge.

dubia, Hook., Bot. Magt. 5,469. Malay Peninsula.

Ord. Begonicza, R. Broien.

Begonia, $L$.

argyrostiga, Fisch., Bruzil.

fuchsioides, Hook, New Grenada.

heraclifulin, Cham. et Schlcht, Mexioo.

hydrocotylifolia, Hook, Mexico.

longifolia, Blume, Java.

nitida, Dryand., Jamaica.

peltata, Otto et Dietr., Javi.

x peltata - stigmosa, Hort., Singapore.

Sambo, Hort.

Saundersii, Hort., Brazil.

semperflorens, $L$. and Otto, Brazil.

stigmosa, Lindl., E. Indies.

undulata, Schot., Rio Janerio.

Rex. Hort., S. America.

Prince Bismarek, Hort.

smaragdina? 
Orev. Cactaces, De Candolle.

Cereus, Haw. peruvianus, Tahern., 8, America. triangularis, Hew, Mexico.

Epiphyllum, Pfeiff. truncatum, Haw., Brazil

Opuntia, Mill. monaeantha, Haw., S. America.

Okd. Usthelifkкa, Juss.

Apium, $L$.

Carum, $L$.

grayeolens, $L$., S. Europe-Celery.

Petroselinum, L., Europe-Parsley.

Fonicnlum, Adans.

vulgare, Gortn, Europe-Fennel.

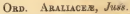

Aralia, $L$.

Guilfoylii, F. Muell., S. S. Islands.

Shepherdiana, Hort., S. S. Islands.

Sieboldii, Hort., S. S. Islands.

Panax, $\boldsymbol{L}$., cochleatum, D. O., Java-Daun mangkok. elegans, $F$. Muell., B. Australia. fruticosum, Link., Moluocas. var. laciniatum.

Fatsia, Deene.

papyrifera, Dene, China-Rice paper-plant.

Brassaia, Endl, Umbrella tree. actinophylla, Endl., E. Australia.

Hedera, $L$., Icy. australis? Australia?

Oro. Connacex, Lindley.

Ancuba, Thumb.

japonica, Thunb., var maculata. Japan.

Ori. Capriflaces, Jusz.

Viburnum, $L$., coriaceum, Bl., Java.

Lonicea,-Honey suckle. chinensis, Wate., China, Japan. 
Ond. Reblack, A. Jusoieu.

Sarcocephalus, $A$ /s.

Leichardtii, F. Muell, E. Australia.

Uncaria, Schreb.

ferruginea, $B l$., Malaya

Gambir, Roxb., Malaya cult., Gambier.

Manettia, Mutis.

cordifolia, Mart., Brazil.

Rondeletia, $\boldsymbol{L}$. odorata, Jacq., Mexico=speciosa, Lodd. odorata + Backhousei, Hort. Kew.

Pentas, Benth. camea, Benth., Africa.

Ophiorhiza, $\boldsymbol{L}$. Mungos, $L$., Ceylon to Java.

Mussenda, $L$, frondosa, $L$, Malaya.

Hamelis, Jacq.

Gandenia, $L$. patens, Jacr., Trop. America.

florida, $L$., China. radicans, Thunb., China, Japan. Stanleyana Hook., Sierra Leone.

Diplospora, D. $O$.

Ixora, $\mathcal{L}$. viridiflora, D. C., Hongkong.

alba, $L$., E. Indies.

Bandhuea, $L$., E. Indies. coccines, $L$, E. Indies. Griffithii, Hook., Singapore. jucunda, Thw., Ceylon.

Pavetta, $L$. rosea, Wall., E. Indies.

Coffea, $L$. indiea, $L$, E. Indies, Singapore. arabica, $L$. Caffa, Abyssinia Coffee. liberica, Heirn., Liberia, Liberian Coffee

Psychotria, $L$. serpens, $L$, Hongkong.

Palicouria, Aubl., = Rhodostoma, Schdie. gardenioides, Schdw., Mexico.

Chasalia, Commers. odorata?

Cephalis, A. Rich, Ipecacuanho. Ipecacusanha, Rich., S. America.

Hydnophytum, Jack. fornicarium, Jack., Singapore. 
Myrmecodia, Jack.

Serissa, Commers.

armata, Jaek., Singapore.

foetida, Commers, China.

" flore pleno.

Orр. Coмrosiт.s, Vaillant.

Elephantopus, Cass.

scaber, $L$., $\mathrm{S}$. America (A naturalized pest in lawns here.)

Mikania, Willd.

Bellis, $L$., Daisy.

Guaco, H. et B., Panama (Reputed cure for snake bite.)

perennis.

Gnaphalium, $L$.

luleo-album, $L_{\text {., }}$ Burope.

Soliago, L., Golden Rod.

Helianthus, $L$. virgaurea, $L_{\text {. }}$, Europe.

tuberosus, L., Brazil, Jernealews Artichoke.

Chrysanthemum, $L$. sinense, Sabin., China.

vars, Horl.

Achilles, $L$.

millefolium, L., Burope, Yarroso.

Stiftia, Mikan.

ehrysantha, Mfikan, Braxil.

Ord. Campanumcex, Barthing.

Centropogon, Prest.

Lucyanus, Hort.

Isotoma, Lindl.

longiflora, Preal., West Indies (noturalized singapor.)

Wahlenbergia, Schrad. grandiflora, Schrod., China.

Ord. Eiricaces, De Canilolle.

Rhododendron, $L$.

javanicum, Ruwdt, Perak, Penang, \&c.

Ond. Plemagringa, Vent.

Plumbago, $L$.

capensis, Thuab., C. G. Hope.

rosen, Lindl, E. Indies.

Oro. Mrasixex, $R$, Brouen,

Ardisia, $L$.

primulaefolia, Gard et Chemp., Hongkong.

solanacea, Roxb., E. Indies. 
Clavija, Ruis ef Pavon.

ornata, D. Don., Caracas.

OrD, Sapotack. Endicher.

Chrysuphyllum, $L$.

Cainito, L, Trop. Ameriea, Stur Ajpit.

Achras, R. Br.

Sapota, I.. Trop. America, Sapodilla, Chiko, chikle Gum

[ tree.

Isomandra, Wivht, Guta pereho.

Gutta, Hoob., Malaya, G. Tubon.

Payenia, D. $\mathscr{O}$.

macrophylla, $D e V v$, Malaya, G. putit, Guitu Sundek.

Mimusops, $L$.

lucida, D. C., Singapore,

Blengi, L., Malaya

Imbriearia, Juss.

Erythroxylon, Boj., Mauritius.

coriacea, Boj., Madagascar.

Ord. Eввкаска, Vent.

Diospyros, $L$.

Ebenum, Retz., Ceylon, Ebony.

quesita, Thw., Ceylon, Calmander.

Ore. Otruckes, Linds.

Jasminum, $L$.

nudiflorum, Lindl., China.

officisale, L., E. Indies.

pubescens, Willd., E. Indies.

Nyctanthes, $L$.

arbor-tristis, L., $\mathbb{E}$. Indies, Java.

Olea, $L$.

fragrans, Thunb. China.

Ligustrum, $I$.

sinense, Lour, China.

rol, var, Hort, Singapore.

Ord, Apoctnaces, Limll.

Allamanda, $L$.

A ubletii, Pohl. S. America.

cathartica, $D . C ., \mathrm{S}$. Ameriea.

neriifolia, Hook., S. Ameriea.

Schottii, Pohl., Braxil.

violaces, Hort.

Willughbeia, Roxb.

edulis, Bond., Malaya.

martabanica, Wall, Malay Peninsula, Gutta Sngyarip. 
Carissa. $L$.

Carandas, L., E. Indies, Malaya.

Melodinus, Forst.

laxiflorus, $B_{4}$, Malaya.

Acokanthera, G. Don.

spectabilis, Benth., S. Africa, =Toxicophlas Sonder.

Alyxia, $R, B r$.

Cerbera, $L$.

sp. Singapore, Gutta jelutong.

Odollam. Gertn., India, Malaya, China.

Thevetia, $L$. nerifolia, Juss, W. Indies (Singapore naturalized.)

Kopsis, Blume.

fruticosa, Roxb., Malaya=Calpiespum Roxburghii, $\theta$.

Vinea, $\boldsymbol{L}$.

rosea, $L$, Mexico.

var, alba.

oculata.

Plumiera, L., Frangipanni. acutifolia, Poir., Trop. America.

Alstonia, $\mathrm{Rr}, \mathrm{Br}$.

constricta, F. Muel., E. Australia.

macrophylla $W a l l$. Singapore.

scholuris, $R$ Br., N. Australia, E. Indies.

Tabernsemontana, $L$.

coronaria, $R . B r .$, E. Indies. flore pleno.

Nerium, L., Oleander.

Oleander, $L$., S. Europe.

Roupellis, Wall.

grata, $W$. et $H_{\text {. }}$. Seirra Leone.

Strophanthus, D. $C$.

surantiacus, Hort., Mauritilis.

longicaudatus, Wight., Singapore.

Urceola, Roxb.

elastica, Roxb., Singapore.

Trachelospermum, Lem,= Rhyncospermum, Lindl. jasminnoices, Lem., China.

Beaumontia," Wall.

fol. var.

multiflora, $T$. et $B$, Java.

Kicksia, Blume.

arborea, Bl., Java.

Ord. Ascherianis, Jacquia.

Calotropis, $\boldsymbol{L}$.

gigantea, $R . B r$, E. Indies, Malay, Mudar. 
Asclepias, $L$.

curassavica, $L$., S. America.

Raphistemma, Wall. pulchellum, Lindl., Moulmein.

Stephanotis, Thouars. floribunda, Brome, Madagasear.

Pergularia, $\boldsymbol{L}$. odoratissima, Aadr, E. Indies,

Hoya, $R . B r$. carnosa, R. Br., Trop, Asia. fraterua, $\boldsymbol{B l}$., Malaya. imperialis, $L$ indl., Malay Peninsula. lacunosa, $B l$, Singapore. major. multiflora, Benth et Hook ॥ Cyrtoceras reflexa.

Dischidia, $R$. Br., nummularia, $\mathrm{R} . \mathrm{Br}_{r}$, Singapore.

Ond. Logaxiacba, Lindley.

Fagrea, Thush.

Gardnerii, Tww., Ceylon. peregrina, $B l$., Sumatra, Temboosie. zeylanica, Thunb., Ceylon. sp. Bukit Timah, Singapore.

Strychnos, $L$.

Tieute, Leschen. Java, Upas Tieute.

Ord. Boragnacex Jussieu.

Heliotropium, $\boldsymbol{L}$. peruvianum, L. var. Hort. Peru.

Symphytum $L$. asperrimum, Bbrst. Caucasus, Prichly Comfrey,

Ord. Cosvolvulackx, DeCandolle.

Ipomæa, $L$.

Horsfalliz, Hook., Sierra Leone.

Learii, Lindl., Ceylon.

reptans, $L$., Malaya. Kankong.

Jacquemontis, Chois.

violacea, Chois. Mexico.

Calonyution, Chois.

speciosum, Chois., Trop. America.

Porana, Burm.

volubilis, Burm., E. Indies.

Oro, Solavaces, Bartling.

Solanum, $L$.

jasminoides, Paxt., S. Americe. macranthum, Dun., Brazil. 
Solandra, Swourt:.

grandiflora, Suarts., Jamaica.

Brugmansia, Pers., Datura, L.

arbore, Pers., S. America.

Knightii, Hort.

Brunsfelsia, L., =Franciscen. Pohl. americana $L$. W. Indies.

Hopeana, Hort.

latifolia, Mikan. Braxil.

ORd. Scrophulakiser, $\mathrm{Br}$.

Angelonia, Humb, and Bonpl.

floribunda, Kune,, Brazil.

Pentstemon, Mitch.

Russelia, Zice.

campanulatus, Willd. 'Mexico.

fuscata?

juncea, Zucc, Mexico.

Uroskimnera, Lindl.

spectabilis, Lindl., Guatemala.

Mazus, Lour.

Pumilio, R. Br., Tasmania.

Orb. Lrxtiausatacax, Lindleg.

Utricularia, $L$.

montana? Columbia.

Ord, Gesnenacex, Erdl.

Gloxinea, L'Her.

vars. Hort.

Achimenes

Chirita ?

vars. Hort.

Tydæa, Dene.

Mdme. Lavalle, Hort.

picta, Decne. \&. America.

vars. Hort.

Gesnera, Mart.

cimnabarina, Hort. S. America.

exoniensis, Hort.

refulgens, Hort.

zebrina, Pact. S. America.

Aschynanthus, Jaek.

javanicus, Hook. Java.

Lobbianus, Hook, Jara.

Chirita, Hamill.

sinensis, Lindl. Hongkong. 
Ord. Bignosiace, $\mathrm{Br}$.

Bignonia, $L$.

Chamberiayuï, sims. Brazil. gracilis, Lodd, S. Ameriea.

Tecoma, Juss. grandiflora, Jacq. do. picta, H. \& B. Guiana

australis, $R . B r$., E. Australia. capensis, R. Br, C. (F. Hope. jasminoides, G. Don, E. Australia. velutina? \&, America

Spathodea, Beave. campanulata, Beave., Trop. Africa. cauaa-feline, Hunce.. Hongkong.

Parmentiera, D. $O$. cereifera Seem., Pamama, Candle tree.

Colea, Bojer. floribunda, Boj, Madagasear.

Crescentia, $L$.

Cujete, L., Trop. America.

ORD. Acaxyace,e, $\boldsymbol{R}$. Brozen.

Thunberyis, L. fil. alata, Hook., Trop. Africa, Singapere naturatized. grandiflora, Roxb., E. Indies. Harrisii, Hook., Madras. laurifolia, Lindl., E. Indies.

Meyeuia, Sehlechld. erecta, Arrab. var. flore alba.

Sanchezia, Ruiz et Pacun. nobilis, $R$. et $P$,, $\mathrm{S}$. America. var. fol. aurea. var.

Acanthus, $L$. ilicifolins, $L$., Singapore.

Barleria, $L$. cerules, Raxb., E Indies. Gibsoni, Dalu., E. Indies.

Crossandra, Salish. infundibuliformis Nees, E. Indies.

Asystasia, Blume. corornandeliana, Nens., E. Indies var. alba.

Eranthemum, $L$. Andersonii, Hook jii. It Todies. bicolor. Srbrawk, Isl. Luzon. fol. var. 
crenulatum, Wall., Malacea, Quedah.

Ecbolium, T, Anders, E. Indies.

macrophyllum, Wall., E. Indies, \&c.

," fol. variegata, Hort., S. S. Islands.

y. versicolor, Hort., 8. S. Islands.

nervosum, $R . B r$., E. Indies, Malacca.

Aphelandra, $\boldsymbol{R} . \boldsymbol{B}$ r.

cristata, $R . B r$., E. Indier.

Fascinator, Lind, et Andr., Brazil.

Justicia, $\boldsymbol{L}$.

Adhatods, Willd, E. Indies.

Rhinacanthus, Nees.

communis, Nees., E. Indies.

Cyrtanthera, Nees.

Pohliana, Nees., S. America.

var. superba.

Bittonia, E., Coem.

argyroneura, Okem, Peru.

rubro nervia, Hort., Peru.

Graptophyllum, Nees.

hortense, Nees., Java.

var, aureo maculatum.

pictum, Nees., E. Indies.

Ord. Verbenacea, Juscien.

Lantana, $L$.

alba, Mill, E. Indies.

mixia, $L$., S. America.

Stachytarpheta, Vahl.

indica, Vahl., Ceylon. E. Indies.

jamaicensis. Vahl., S. America.

mutabilis, $\mid a h l$, Trinidad.

Duranta, $L$.

Plumierii, Jacq., S. America.

flore alba.

Callicarpa, $R_{a x b}$.

lanceolaria, Roeb., Hongkong.

Tectona, $L$.

grandis, L., N. Indies. Teak.

Faradaya, F. Muell.

pepuana, $F$. Muell., New Guinea.

splendida, $\boldsymbol{F}$. Mveller., N. F. Australia,

Clerodendron, $L$.

acuminatum, Wall., Singapore.

fragrans, Willd, Japan.

,3. fleno.

infortunatum, $L_{.,}$E. Tndies.

longicolle, F. W. Neyer., Guiana. 
nutans, Will, E. Indies.

Thompsone, Balf., Afriea.

var. Balfouri, Hort.

viseosum? Ceylon.

Petriea, L.

volubilis, L., S, America.

Homskioldia, Rets.

sanguinea, Retz, Java.

OrD. Lishints, Juss.

Coleus, Lour.

aromatiens, Benth., E. Indies.

pictus, Hort., Duke of York Island.

Salviat, $I$.

numerons garden vars, and seedlings.

eoccinea, L., Horida.

splendens, Ker., Brazil.

Oni. Plaxtagixya; $R, B r$.

Plantago, L.

major, L., Enrope.

Ord. Nrotaeivief, A. Juss.

Mirabilis, $I$.

Jalapa, L., Peru. Marvel of Pera.

-Bongainvillea, Comm.

spectabilis, Hook., S. America.

var. glabra.

, speciosa.

Ond. Phytolaccacea, R. Bromen.

Rivinin, $L$.

humilis, L., E. Indies.

Petiveria, $L$.

alliacea, L., Mexico.

var, octandra $M i q$.

Ord. PoLyoones, Jersieil.

Coccoloba, Jacq.

platyclada, F. Muell., E. Australia.

Antigonum, Endl.

leptopus, Hook., S. America.

Polygonum, $L$.

capitatum, Don., Nepal.

Ord. Amarastadia, R. Brown.

Iresine, Willd.

Herbstii, Hook, Brazil.

var, aureo-reticulata. 
Alternanthera, Forsk.

versicolor, Hort.

Ond. Chzmopones, $\boldsymbol{R}$. Brown.

Rhagodia, R.Br. Salt Bush.

hastati, $R . B r_{t,}$ Queensland.

parabolica, $R . B r$, do.

Ord. Baszluacre.

Basella, $L$.

alba, $L$, E. Indies. Java.

Orp. Mrristicicea, Lindley.

Myristica, $L$.

fragrans, Houst., Moluceas. Nutneg.

var. macis-albis. White maced. do.

fatua, Houtt., Molueeas, Long Nutmeg.

Orv. Laurixea, DeCandollo.

Cinnamomum, Burm.

aromaticum, Burm. Chins, \&ee.

Camphora, Nees, China, Japan. Camphor.

zeylanieum, Nees., Ceylon. Cinnamon.

Persea, Grin.

gratissima, Grfn., W. Indies. Alligator, or Avoeedo Pear.

Eusideroxylon, $T$, et $B$.

Zwagerii, $T$. et B.. Japa.

ORd. Thronzes, Adanson.

Drimyspermum. Reine.

Burmanni, Bl. Jave.

urens, Reinw. do.

Wickstræmia, Endl.

indica. E. Indies.

Ord. Protracri, R. Brown.

Macadamis, $F$, Muell.

ternifolia, F. Muell., N. Australia. Queensland nut.

Grevilles, $R$. $B r$.

Banksii, R. Br., N. A ustralia.

Hillians, $F$. Aruell., Australia.

robusta, Cisnn., E. Aus ralia.

Hakea, Schrad.

levicoptera, R. Br., W, Australia. 
multilinenta, var. grammatophylls Meisn. S. Australia. saligna, Kright., \$. Australia.

Buckinghamia, F. Muell. celsissima, F. Muell., N. Australia.

Stenocarpus, $R$. Br.

Cunninghamii, $R . B r$, N. Australia.

salignus, R. Bc., E. Australia.

Ond. Unticex, R. Br.

Laportea, Gaudich.

Pilea, Lindl.

Schomburgkii versicolor, Bull, S. S. Islands.

muscosa, Wedd., Trop. America.

Behmeria, Jacq. nivea, Hook, Ohina. Rhea fibre, Rameh.

\section{Ord. Mgaxis, Endlicher.}

Brosimum, Sicartz.

Alicastrum, \&uts., Jamaica. Bread nut.

Artocarpus, $L$.

Blumei, Thec., Java, Borneo. Tarrap.

echinatus? Malaya. Monkey Jack.

incisa, L. jll., S. S. Islands. Bread fruit.

iutegrifolia, L., E. Indies. Jack fruit.

polyphema, Pers., Malaya. Tjampedah.

rigida, $B l$., Java, \&e. Tompang.

Castilloa, Cerv.

var. glsbra, Murt. Singapore.

elastica, Cerv., Cent. America. Panama Rubber.

Ficus, L. Fig tree.

australis, Willd., E. Australia.

benghalensis, $L_{*}, \mathrm{~B}$. Indies,

Cannonï, Murt, N. Australia=Artocarpus, Hill.

Carica, L., S, Europe. Common Fig.

Cunninghamii. Miq. N. Australia.

diversifolius? Singapore.

elastica, Razb., E. Indies, Malay Pen., India Rubber.

hirta, Miq., Malays

indica, $L$., E. Indies.

macrophylla, Def., i. Australia.

pendula, L., Malaya

polycarps, Roob., Malays

racemifera, Roxb., Malaya

rapiformis, Roxb., Malaya

religiosa, L., E. Indies. Prepul.

retusa, Miq., Jars. Waringan.

stipulats, Thunb, China. 


\section{8}

Maclura, Mott. Osage Orange.

aurantiaca, Mett. N. America.

Ord. Centrides, Endlicher.

Girroniera, Gaud.

subequalis, Planch. Singapore.

Ord. Casuarming, Mirbel.

Casuarina, $L$.

equisetifolia, Forst., E. Indies.

stricta, Ait., Australia.

sumatrann, Jungh. Sumatra.

tephrosperma, Hort. Hamb. S. W. Australia.

torulosa, At, B. Australis.

Ord. Everhobiaces, $R$, Brozon,

Dissiliaria, F. Muell.

Reidia.

baloghioides, F. Muell. N. Agstraliat.

Croton, $L$.

glaucescens. Singapore.

laceiferum, $L_{4}, \mathrm{E}$. Indies.

Tiglium, $L_{v}$, do.

$\mathrm{V}$ ars., Hort.

angustifolium.

ancubrefolium.

aureo-pheniceum.

aureo-spirale.

aureo-strictum.

cornutum.

elegans.

Hendersonii.

interruptura.

irregulare.

Macenfeeana.

maculatum-Katani, MoArthur. New Guines.

maximum.

pictum.

picturatus.

spirale.

tortile.

trilobus.

y var. Albertii.

" $"$ Dismeli.

" 3 Lord Cairns.

$y \quad y$ Susan Rmmeline.

undulatum.

Traveller.

rariegatum. 
Hevea, $A w b l$.

braziliensis, Mull., Brazil. Brasil rubler.

Baloghis, Endl.

lucida, Kindl. Norfolk Island.

Aleurites, Forst.

triloba, Forat. Ceylon Malaya

Acelypha, $L$.

Macafeesna, Hort., S. S. Islands.

marginata, $\mathrm{Spr}_{\text {, }}$ do.

tricclor, Hort. do.

Manihot, Plem.

Glaxiovi, Hook fil., Central America. Ceara Serap rubber.

Jatropha, $L$. utillissima, Pohll, S. America. Cassarn.

Carcas, L., S. America. Physic nut.

multifida, $L_{2}, \mathrm{~S}$. America.

pandurefolia, Andr., Guba.

par, roset.

podngrica,
Stillingia, Gordn. podegrica, Hook., s. America.

sebifera, Miehs., China, Chinese tallow tren.

Dalechampia, Plum.

Roesliana, A. Mrell. Mexico.

var, rosea.

Euphorbia, $\boldsymbol{L}$.

antiquorum, $L_{.}$, E. Indies. Malsya

Bojerii, Hook, Madagascar.

splendens, Bojer.t. do.

Tiruenlli, L., E. Indies, Malaya. Phillipines.

Antidesma, Burm.

Dalladyana. F. Muell., E. Australis. Herbert-Vale Cherrg.

Poinscttia, Graham.

pulcherrims, Grah., Mexico.

Pedilanthus, Neck.

tithymaloides, Port., 8. America.

Ord. Nepgntaks, Bhrme.

Vepenthes, L., Pitcher Plant.

albo-marginats, Lobb., Penang, Perak.

ampnllacia, Jack., Singapore, \&c.

var. vittata. Singapore.

destillatoria, ' $L$, Ceylon.

gracilis, Korth., singapore.

Kennedyana, F. Micell., Cape York.

levis, Jack., Singapore.

melamphora, Rne., Java. phyllamphora, Wild., Mount Ophir.

Rafflesiana, Jack., Singapore. 
var. giaberrima, Singapore. sanguinea, Lindl., Perak. Mount Ophir.

Ord. Prpenaces, Bndlicher.

Piper, $L$.

Betle, L., E. Indies. Betel.

Cubeba, $L$, fil, Java. Cubebs.

nigrum, $L_{*}$, E. Indies. Pepper.

Chavica, Miq.

Roxburghï, Miq., E. Indies=Piper longum, L.

Artanthe, Miq.

elongata, Miq., Peru. Matico.

Peperomia, ficiz et. Pav., Pavon.

arifolia, Mig., Brazil.

Ord. Chioranthaces, Lindl.

Chloranthus, $S w$.

inconspicaus, $S_{v}$. Japan.

Ord. Cosirenes. Vent.

A rancaria, Henk et Hochst.

Bidwillii, Hook., E. Anstralia. Bunza.

Cookii, $R . B r$. , New Cajedonia.

Cunninghamii Ait. Movetor Bay Pine.

var. glanea.

excelsa, R. Br., Norfolt Island Pine.

Rulei, F. Muell., New Caledonia.

Dammara, Rumph.

orientalis, Hook fil, Bormeo.

anstralis, Lam., New Zeeland.

ovata, C. Moore,, New Caledonis.

robusta, O. Moore, E. Australia.

Cryptomeria, Don.

Biota, Endl.

japonica, Don., Japan.

orientalis, Endl., China.

Cupressus. Touref

funebris, Endl., China. Feneral Oypress,

tornlosa, Don., Himslnyas.

var, elegaus.

Dacrydium, Soland.

Horsfieldii? Penang, Perak, $2-3,000 \mathrm{ft}$.

Podoearpns, L. Heril.

australis, Lam., Australia.

cupressina, $R$. Br., Malaya 2,500 to $3,000 \mathrm{ft}$.

potystachyo, R. Br, Singapore, Penang.

spinulosa, $R$. Br., Australie. 


\section{Okd. Crcadse, Persoon.}

Cycas, $L$.

circinalis, L., E. Indies. Malaya

media, R. Br., E. Australia.

Normanbyana, $F$. Muell., E. Australia. revoluta, Thunb, Japan.

Macrozamia, Mig.

cylindriea, F. Muell., Queensland.

Miquellïi, F. Muell., N. Australia.

Mekenz'ei, F. Muell, do.

MeKenzet, F. Muell, $=$ Dennisonii.

Peroffskyana, Miq, N. Australis.

sclerocarpa?

spiralis, $t$. Muell., N. Austratia.

teninfolis? Queensland.

Catakidozamia, F. Muell.

Hopei, F. Muell. Queensland.

Bowenia, F. Muell.

spectabilis, F. Muell., N. E. Australia.

var. serrulata.

\section{ENDOGENS.}

Ond. Caxnacix, Agardh.

Canna, $L$.

indica, $L_{v}$, E. Iudies. Indian Shot.

Maranta, $L$.

arundinacea, L., S. America. Arroukroot.

Bellula, Hort.

discolor, Hort.

fasciate, Hort.

illustris, Lind., S. America.

Legrelliana, Hort.

Lindenii, Andr., Peru.

Maksyana, E. Morr. S. America.

micans, Hort.

Porteana, Hort, S. America.

Warcewiczii, Lind., S. America.

Zebrina, S. America.

Phrynium, Willd.

sanguineum, Hook., S. America.

Ord. Zrvorgenaces, L. C. Richards.

Globba, $L$.

aurantiace, Miq., Sumatra and Perak.

leucantha, Mig., do. do. 
Blettaria, Rheede:

Cardamonnum, White., Ceylon cult. Cardamum.

Zingiber, $L$.

officinale, Rox., India, Ginger.

Zerumbet, Rox., India.

Alpinia, $L$.

cernua, Sixus., E. Indies.

Galanga, Sw., Malaya

magnifica, Rox., Madagascar.

nutuns, Row. E. Indies.

Hedychium, Krenig.

angustifolium, Roxb., Bexgad.

coronarimm, Koen., India.

var-flavescent., Java.

Amomum, $L$.

Melegueta, $R \boldsymbol{s}^{0} b$. India.

pulchelluw, The., Ceylon.

Kampferia, $L$.

rotundata, $L$., E. Indies.

Cureuma, $L$. speciose, Hort.

anstralis, Australia.

Costus, $L$.

longa, $L$, E. Indies, \&ec, Thrmeric.

speciosus, Sin,, India.

Ord. Mesacka, Agardh.

Heliconia, $L$.

* bicolor, Kltsch, Brasil.

Musa, $L$, Plaintarin, Barana.

Cavendishii, Paxt., China.

coccinea, Andr., China.

paradisiaca, L., E. Indies, vars, cult.

rosacee, Jacq., Mauritius.

sapientum, L., E. Indies, vars. cult.

superba, Roab., S. India.

Strelitga, Banks. textilis, Nee, Phillipine Islands. Manilla Hemp.

juncea, Andr., C. G. Hope.

ovata, Don., C. G. Hope.

Reginæ, Ait., O. G. Hope.

Ravenala, Adans., Traveller's Tree.

madagaseariensis, Sonner, Madagascar.

Ond, Bemelacex, Lindley.

Ananassa, Mill.

sativa, Mill, Pine Apple.

var, fol. variegata. 
Eehmen, Ruis et Pav.

fulgens, Melin., Braril.

\section{Ord. Orchings, Br.}

Oberonia, Lindl.

iridifolia, Lindl., E. Indies.

Microstylis Nust.

congesta, Rchb. fll. Hongkong.

discolor, Lindl., Ceylon.

Rheedii, Landl, do.

Dendrobium, Swo.

aduncum, Lindl, E. Indies.

agrregatum, Roxb., E. Indies.

albo-sanguineum, Lindl., E. Indies.

albux! Lindl., E. Indies.

sincenum, Wall., Nepal.

aureum, Lindl, E. Indies.

barbatulum, Lindl., $\mathbb{E}$. Indies.

Bensonis, Rehb. fll., Moulmein.

Boxallii, Rchb. fu, Assam.

Bullerismum, $R_{c h} b_{+} f i l$, E. Indies.

bigibbum, Lindl., N. Australia.

Calceolus, Hooker., E. Indies.

Cambridgeanum, Livdl., Chittagong

canaliculatum, $\boldsymbol{R}$. Br., Australia.

ohlorops, Lindl., E. Indies.

chrysanthum, Wall., Nepal.

ohrysotoxum, Lindl, Nepal.

ciliatum.

clavatum, Paxt, E. Indies.

compressum.

crassinode, Rebb. fll, Siam.

orepidatum, Hook., Burma.

B. roseum.

cretaceum, Lindl., Burma.

crystallinum, $R$ chb. $f \mathrm{l}$, Arracan Mts.

crumenatum, Swartz., Malaya

B. majus.

cucumerinum, Lindl, Malaya.

Dayaunm Rchb. fl., Borneo.

Delhousianum, Paxt., E. Indies.

densiflorum, Wall, E. Indies.

Devonianum, Paxt., E. Indies.

cburneum, Rchb. f., Moulmein.

excavatum, $\boldsymbol{B l}$., Jeva.

Faleonerii, Rehb., D. Indies.

Farmerii, Paxt., E. Indies.

fimbriatum, Hook., Nepal. 
B. oculatum, Nepal.

Fitzalanii, F, Muell., Australia.

Fitzgeraldii, $F$. Muell., do.

formosum, Lindl., E. Indies.

giganteum, Rangoon \& Moulmoin

Fytchianum, Rangoon.

glaucophyllum, $\boldsymbol{T}$. et $B$., Java.

Gibsonii, Paxt., E. Indies.

Griffithianum, Lindl., E. Indies.

heterocarpum, Wall, Nepal.

Hillii, $F$. Mrvell., Australia.

Jenkinsii, Lindl., Gualpara.

Johannis, Australia.

Kingeanum, Australia.

linguzeforne, Svoartz., Australia.

longicorme, Lindl., E. Indies.

luteolum, Rehb. t., Moulmem.

macranthum, Lindl., Amboina. giganteum, Manilla.

macrostachyum, Lixdl., E. Indies.

McCarthis, 7 hw., Ceylon.

moschatum, Wall., E. Indies.

'mutabile, Lindl., Java.

nobile, Lindl., Macao.

nodatum, Rchb. $f$., Moulmein.

Parishii, Rehb. f., Moulmein.

Pierardii, Roxb., Ganges delta.

B. majus.

primulinum, $\boldsymbol{R} c h b$., E. Indies.

rhododypterum, Rell ... Assam.

rigidum, $B$ l., Java.

salicornioides, $T$. et B., Pakata.

sanguinolentum, Lisdl., Bombay.

B. sureum.

secundum, Lindl, Malaya.

speciosum, 8 m., Australia.

suavissimum, Rchb. f., Burmah.

striolatum, Australia.

Sumnerii, F. Muell., New Guinea.

superbiens, $F$ : Muell., do.

tortile, Rehb. $f_{+}$, Burmal.

thyrsiflorum, Rchb. f., E. Indies.

transparens, Lindl., Vi. Indies.

undalatum, Brown., Australis.

Veitcheanum, Lindl., Java.

Wardianum, Rchb. f., Assam.

Lowii, do.

Zollingerianum, $T$. ot B., Javu and Singapose.

B. album, Singapore. 
Polystachya, Hooker.

Lria, Lindl.

grandiflora, Rehb. f, Mexico.

convallarioides, Lindl., Nepal.

flava, Losdl., Java.

fusco-viride.

multiflora, Lindl, Malay Peuinsula.

planicanlis, E. Indies.

vestita, $H_{00}$., Malaya.

Aporum, Blume.

anceps, Limll., B. Inties.

indivisum, Bhme., Java.

Bolbophyllum.

acicunium ?

Baileyii, Australia.

deletescens?

raculatum, Rchb., Moulmein.

purpureum, Java.

sp. Seychelles.

spp. Malay w.

Cirrhopetaium, Lindl.

Medusse, Lindl, Borneo.

Paludii de $V_{r}$. Java.

stramineum, $T$, ef $B$., Sumatra.

Thouersii, Lindl, Java.

spp. Malaya.

Cologyne, Lindl.

asperata, Lindl.,-Lowï, Paxt. Borneo.

cinnamonea, $T$, et B., Java.

Cummingii, Lindl, Palembang.

corrugata, Wight., Khasya.

fimbriata, Lindl., do.

flaceida, Lindl., Nepal.

longifolia, Landl., Mt. Salak.

longipes, Lindl., Khasya..

ocellata, Lindl, , E. Indies.

ochracea, Lindl, Khasya.

ovalis, Lindl, Kumaon.

pendurate, Lindl., Borneo.

Parishü, Reȟb, f., Moulmein.

specioss, Lindl., Java.

testacea, Lindl., Singapore.

spp. Malaya.

Pholidota, Lindl.

articulata, Livdl., E. Indies.

imbricata, Lindl., E. Indies. Malaya. 
Epidendrum, $L$.

ciliare, $L$, 8 . Ameriea.

selligerum, Batem., Guatemala. purpeneum.

Cattleya, Lindl. citrina, Lindl., Oasaca.

Dowians, Reȟb. f., Costa Rica.

Mossiz, Lindl., La Guayra.

Skinneri, Batem., Costa Rica. B. rosea.

Schomburgkia, Lindl.

gloriosa, Rehb. $f$., S. Ameriea.

Brassavola, Lindl.

undulata, Lindl., La Guayra.

Digbyana, Honduras.

glauca, Liadl, Mexico.

nodnss, Lindl., Jamaica.

Phaius, Lindl.

Bernaysiana, Roab., N. Australia.

bicolor, Lindl., Ceylon.

callosus, Liwell., Java.

grandifolius, Lour, Perak. China.

luridus, Thw., Ceylon.

maculatus, Lindt, E. Indies.

Wallichii, Lisell., E. Indies.

Arundina, Bl.

bambusefolia, Lindl., E. Indies.

chinensis, $B l$, China.

denss.

speciosa, $B l$., Java.

Spathoglottis, $B$ l.

Fortunii, Lindl., Hongkong.

plicata, Bl., Singapore. Malaya.

Collabium, $B l$.

nebulosum, $B l$, Java.

Plocoglottis, $B$ l.

acuminata, $B l$, Singapore.

javanica, Bl, Perak, Johore.

Eulophia, R. Br.

macrostachys, Lindl., E. Indies, Singapore. virens, Lindll, $\mathrm{D}$. Indies.

Cyrtopera, Lindl.

Gandnerii, Thac, Ceylon.

Zygopetalum, Hook.

Mackayii, Hk., Brazil.

Sarcanthus, Lindl.

teretifolius, Lindl., China.

Houlettia, Brogn.

Brocklehunistian, Litidl., Brazil. 
Phalsenopsis, Blome.

amabilis, Blume., Manilla.

grandiflora, Lindl., Tambilau Islands.

auree, Borneo.

cornucervi, $K$. et $H$, Javs, Malsy Peninsula.=Polychilus.

Lowii, Rchb. $f$, Moulmein.

Luddemanniana, Rchb., Phillipines.

Parishii, Rehb. f, Moulmein. roses, Lindl., Manilla.

Schilleriana, $R \cdot h b$., Phillipines.

sumatrana, $R$ s hb., Sumatra.

violaces, $T$. et $\dot{B}$., Pontianak.

var. Murtoniana, $R c h b$. $t$, Kinta.

sp. nova. Pontianak.

Bromheadia, Lindl.

Finlaysoniana, Reichb, Singapore.

Vanda, R. Br.

Batemanni, Lindl, Phillipines.

ccerulea, Griffih., Khasia.

gigantea, Lindl., Moulmein.

Hookerii, Labunn, Kinta.

insignis, (true) Blame, Timor.

lamellosa, Manilla.

limbata, BTwac., Java.

lissoehiloides, Mamilla.

Lowii, Lludi., (Renanthera), Borneo,

Roxburghii, $R$. $B r$, E. Indies.

var. corrulea.

spathulata, Spreng., Ceylon, Mysore.

suavis, Lindl., Java.

teres, Lindl., Burmah.

tricolor, Lindl., Java.

Renanthera, Lour.

arachnites, Lindl., Java.

var, rosea.

coccinea, Lour, China.

Saccolabium, Lindl.

ampulisseum, Lindl, B. Indies. -

Blumei, Lindl, E. Indios, Java, Phillipines.

curvifolium, Lindl., Nepal.

giganteum, Lindl, E. Indies.

guttatum, Lindl., B. Indies.

Harrisonii, $R c h b$, Pulo Copang.

var. violacea,
Hendersonianum, Rchb. $f_{2,}$ "Pontianak.

obliquum, Lindl, B. Indies.

paniculatam.

roseum, Lindl., E. Indies.

rubrum, Lindl., $\mathbf{E}$. Indies. 
Cleisostoma, Blume.

Fordii, Hance, Hongkong.

Stanhopea, Lindl.

eburnea, Rchb., Braxil.

Frides, Lowr.

affine, Wall, E. Indies.

var, roseum, Lankives.

Huttonii, Hook. f, Borneo.

Tindleyana. E. Indies.

maculosum, E. Indies.

odoratum Lour, F. Iudies.

quinquevulnerum, Lixdl., Phillipines.

suarissimum, Th, Lobb., Borne-

suaveoleus, $B l$, Java.

testaceum, Lindl., E. Indies.

Teysmannianum, Miq,-, Java.

virens, Maloya.

Wightianum, Linill., E. Indies.

sp. nova, flore rosea. Soloo Islands

Calauthe.

angustifolia, Liad?, Gumong Hijnn. Perak, 2,500 feet.

eurculigoides, Lindl. . " , , 2,000-2,500 ft.

veratrifolia, R. Br., Nilgherries to Anstralia.

vereicolor, Lindl., Ceylon.

Thelasis, $B$ l.

vestita, Lindl, Burmah and Borneo.

capitata, Bl., Javi.

earinata, Bl., Java.

Miltonia, Lindl.

- Cloweaii, Lindl., Brazil.

Limatodes, Blume.

rosea, Lindl., Moulmein.

Oncidium, Swartz.

altissimum, Swarlz., S. America.

ampliatum, Lindl., C. America.

majus.

fiexuosum, Sims., Brazil.

luridum, Lindl., W. Indies.

pictum, H. B. K., Peru.

sphacelatum, Lindl., Guatemala.

majus.

Brassia, R. Br.

maculata, $R$. Br., Jamaica.

Wraye, Lindl., Brazil.

Lycaste, Lindl.

cruenta, Liadl., Guatemalu.

Harrisonii, Lindl., Brazil. 
Angreoum, $P$. Thown

eburneum, Thowars., Madagascar.

gladiifolinm, Thouars., Mauritins.

sesquipedale, Hook. Madagascar.

Trichopilia, Lindl.

saperbum, Thovors., Madagascar.

coccinea, Lindl., Costa Kica

Cymbidinm, Swarts.

aloifolium, Sw., E. Indies. Malaya,

var. album.

bieolor, Livill., 15. Inlies.

canaliculatum, Broven., Australia.

ensifolium, Siv., Japan, E. Indies

eburneum, Hook., K. Indies.

trieolor, Scheff," Japai.

sangainoum, $T$. , ef $B$., Java.

Thrixpermum, Lorr.

unguiculatum, Relib., Manilla (a warthless sp, often sold for

Phalenopsis rosea.

Grammatophyllum, Bhume.

multiflorm, Manilla.

scriptum, Bi., Amboina.

speciosmm, B/., Malaya.

Jeopardanthus, $B$ i.

scandens, $B /$, Java, Singapore.

Auguloa, $R$. et $P$.

Clowesit, Liudl, Columbia.

Acampe, Lindl.

multiflora, Lindl., Chiua.

Wightiana, Lmelh, E. Indies.

Habenaria, $W$ illd.

Susanna, $B r$, R. Indies. Hongkong.

Vinilla, Sarz.

aromatica, Sw., Trup. America, Vanilla.

sp. Singapore.

Goodyera, Lindl.

procera, Hook., Singapore.

Anactochilas, Blame.

Dawsonianus, Low. Malaya.

Lowii, Hort., Borneo.

setaceus, Bi., Ceylon. Mistays

xanthophyllus, How. frinong Pnlai, Johor

Cypripedium, L., Lady'x Slipper.

barbatum, Lisid., l'onang, Quciali.

Boxallii, Rchib., Assana.

concolor, Hook, Moalmein.

Hookera, Lindl, Java.

Lawrenceanum, Rchb, $t_{*}$, Borneo.

Lowii, Lindl, Borneo. 
niveum, Rehb., Lankives.

purpuratum, Lindl., Hongkong.

Stonei, Hook, Borneo.

Ord. Aposmasiaca, Blume.

Nenwiedia Bhome,

veratrifolia, Bl., Java.

\section{Oep. Tsecices, Lindl.}

Ataccia, Prest.

cristata, Landl., Mslay Peninsula. Singmporc.

OxD. ImIDKR.

Pardanthus, $K$ er,

chinensis, Ker., China.

Tritonia, $K e r$.

Ixia. $L$.

aurea, Ker. O. G. Hope.

ameua, Link.

\section{Vars. Hort.}

Iris, $L$.

speculatrix, Baker., Hongkong.

ORD. Amarinidex, Br.

Clivia, Lindl.

nobilis, Lindl, S. Afrien.

Imantophyllum, Hook. ministum, Hook, C. G. Hope.

Doryenthes, $R$. Br excelsa, $R: B r$., E. Australin.

Palmerii, Hill, N. Anstralia.

Agave, $L$. American dlos. umericans, $L$, Mexico.

Fourcroya, Vent.

gigantea, Vent., S. America.

Amaryllis, $L$.

Belladonna, L., C. G. Hope. Has wot yet ploatered thre.

Hippeastrum, Herh., numernus seedling nars. 
ignescens, Sweet., Hybr.

Zephyranthes, Herb.

rosea, Lindl., Cubs.

Vallota, Herb.

purpurea, Herb., C. G. Hope.

var. miniato.

Eucharis, Lindl.

13. oculeta.

amazomica, Lindl., Amazon.

Alatromeria, $L$.

aurea, Grah., Chili.

hraziliensiB, Sellon., Brazil.

Ginum, $L$.

amnbile, Donn., Sumntrn.

asiaticum, $L$., China, N. Australia.

oruatum, Herb., B. Indies.

Eurycles, Saliab.

pedunculatum, $R$. Br., S. Australia.

australis, Ait, Anstralia,

Cunningbamil, Mit., Anatralia.

Paneratium.

biflorum, Roxb., Hongkong.

malabaricum, $H \in r b$., E. Indies.

speciosum, Salisb., W. Indies.

ORD. Hyroxides, $B r$.

Cureuligo, Grta.

recuravata, Dryand, Singapore,

Ond. Droscoske, $\mathrm{Br}$.

Dioseorea, $L$.

illustrata, Lind, Rio Grande.

Ord. Pangex, Mart.

Seaforthia, $R, B r$.

Areca, $L$.

elegans, $R$, Br., E. Anstralia.

alba, Bory., Madngascar.

aurea, Hort., Seychelles.

Bauerii, Hook., Norfuld Islanit.

Catechu, L., E. Indies, Maslaya Betel nut, Pinang.

var, alba.

") trang buinn.

crinita, Bory., Mauritius. 
concinna, Thew., Ceylon.

lutescens, Bory., Mauritius. monostachya, Mart., N. Australia.

Nenga, Blume,, Java.

Normanbyann. purpurea.

rubra, Bory., Madagascar. sapida, Forst., New Zealand. triandra, Raxb., E. Indies.

Hyophorbe, Grtn.

amaricaulis, Mart., Bourbon.

indica, Gertn,, Bourbon.

Oreodoxa, Willd, ( Dabbage Palm.)

oleracen, Mart., Antilles.

regia, H. B K., $\mathrm{S}$. America.

Pinanga, Blume.

maculata, Porter., Perak, Qnedah. ternatensis, Tsland Ternate.

Kentia, Bluse.

Belmoreana, C. Moore, Howes' Isirnd.

Canterburyana, C. Moore, , , ,

Forsteriana, C. Moore.,

Moorei, F, Muell.

MeArthuri, Hort. Veitch, New Guinea.

$?=$ robusta, $P=$ Cyphokentia, Lind., New Guinea.

Wendlandtians ?

Orania, Griff.

macrocladus, Griff., Singapore. (Ehonl.)

Caryota, $L$.

Cummingii, Hook., Singapore.

obtusa, Griff., Singapore.

Rumphiana, Mast, New Guinea.

sobolifera, Wall., Singapore.

arens, L., E. Indies, Malayn.

Wightiana, Thw., Ceylon.

Loxococeus, = Ptychospermn, Ther, rupicols, Ceylon.

Ptychosperma, Labill.

Álexandræ, F. Muell, N. Australis.

Calapparia, Beccari, Malaya.

singaporensis, Beccari, Siigapore.

coccinea, H. L. B., Malaya.

Arenga; Labill.

obtusifolia, Mart., Malaya. saccharifere, Lahill., Malaya.

Wallichia, Roxh.

caryotoides, Roxh, E. Indies.

Dypeis, Noronh.

sp. Madagasear. 
Oncosperma, Blume.

fasciculata, Ther, Ceylon.

Calyptroealyx, Blume.

spicatus, Bl., Banka.

Calamus, $L$.

anstrulis, Mart., N. Australia.

ciliaris, Blume., Malaya.

Roxburghii, Griff., B. Indies,

rudentum, Roxb., E. Indies.

tenuis, Rod., E. Indies.

Plectocomia, Rrut.

elongats, Bhume., Singapore.

Zalaces, Rnse.

edulis, Reinwe, Singapore. Salak.

Calamosagus, Griff.

spp. Singapore.

Eugeissonia, Griff.

Sagus. Rumph.

triste, Griff., Malny Peninsula. (Bertem.)

lavis, Rumph., Moluceas.

Licualn, Rusuph.

Melay Pawiner. horrida.

longipes, Griff., Singapore.

Sabal, Adara. peltafa, Roxb., E. Indies.

Adausonii, Guerns., Mexico.

longipedunculsta, Hort. $H, \&$ \&.

mexicana, $\boldsymbol{H}$. \& Schmidt.

princeps, H. \&. Schmidt.

minor.

Palmetto.

umbraculifera, Mart, Jamaica

Chamerops, $L$.

Fortunei, Hook, China.

Trithrinax, Mart.

mauritireformis, Hrt., Venezuela.

Rhapis, L. fil.

flabelliformis, L. fil., China.

Thrinax, L. fil.

Phomix, $t$.

parviflora, Jamaica.

acaulis, Ham, E. Indics.

canariensis, Hort. H. \& $\mathcal{S}$.

dactylifera, L., Egypt. Date Palm.

paludosa, Roab., Malay Peninsula.

sylvestris, Roxb., E. Indies.

Phonieophorium=Stevensonia.

seychellarum, Seychelles. 
Acrocomia, Mart.

sclerocarps, Mart., Brasil.

Attalea, $\boldsymbol{H}$, et $\boldsymbol{K}$.

Cohune, Jamaica.

Martinezia, $H$. ef $K$.

caryotafolia, $H$. et $K_{\text {, }}$, Peru.

granatensis, Hort. Bull., N. Grensda.

Cocos, $L$.

flexucse, Mart., Brazil.

nucifera, $L$., Cocoanut. Klappa, MaI,

var, $K$, gading., Mal. nom. vulg.

"n logie ,

" poojie $n=$ =rar. pomila. Hasskl.

" $"$ longehong.

"pulat.

$\rightarrow$ Rojah.

Saboot putih.

plumosa, Lodd., W. Indies.

Elweis, Jacq., (African oil Palur.)

guineensis, Jacg, W. Africa

Jubiea, $H$. el $K$.

spectabilis, $H$, of $K$, Chili.

Phytelephas, Ruiz. et Pavon,

macrocarpa, $R_{\text {, et }} P$., Peru (Vegetable Ivory Nut.)

Nipa, Rumph.

fruticans, Wurmb, India, Malaya.

Orb. Panbanex, Br.

Pandanus.

humilis, Rumph., Singapore.

Lais, Kurz., Benka.

utilis, Bory., Madagasear.

Ord. Crolanmina, Poiteau.

Carludovica, $R$. et $P$.

palmata, $R$. et $P$., N. Grenada, (Panama-hat palm.)

Ord. Frfxcixemes, Brong.

Freycineta, Gaud.

angustifolia, Bl, Malaya.

OtD. AROIDEN.

Aglaonemn, Schalt.

nitidum, $\boldsymbol{K}^{t} \boldsymbol{h}$., Singapore and Penang. 
Alocasis, Schott.

cuprea, C. Koch., Borneo=metallica, Hook.

indiea, Sch., India.

Jeuningsii, Veitch., India.

Lowii, Hook., Borneo, Perak.

macrorhiza, Sch., India, Polynesia.

fol. var.

Marshallii, Bull, India.

singaporensis, Lind, Cat., Singapore.

Veitebii, Schott., Perak.

Anthurium, Sihott.

digitatum, Kth., Veneruels.

Arum, $L$. divaricatam, L., Hongkong, B. Indies. trilobatum, L., Ceylon.

Caladium, Vent. amabile, Hort. argyrites, Versch, 8. America. argyrophyllum, Hort.

Alfred Bleu, Hort.

Auber, Hort.

Barillet, ,

Baraquinit, Hort.

Beethoven, $n$

Brogniartii.

bicolor, Vent, Brazil.

Burrel, Hort.

Baron Humboldt., Hort.

Cannærtii, Hort.

Chantinii, Lem., Braxil.

Ducharto, Hort.

Dr. Lindley, ,

Duc de Malakotf, Hort.

Duc de Ratisbon, "

E. G. Henderson, "

Houlletii,

Jules, Putreys, is

Lepeschkinei, "

Louis Poirier,

Luey,

marmoratum,

Max. Kolb,

mirabile,

Meyerbeer,

Mrs, Dombrain.

Mdm. Andreux.

Napoleon III., Hort.

Newmannii, 
Prince Albert Edward, Hort.

Princess Alexandra,

Petschkinei

picturatum,

Poecile, sch., Brazil.

Pennierii, Hort.

regale,

Reine Vietoria, Horl.

Ricei,

Rossini,

rubricaule,

Sehwelerii, Hort., Para.

Sinitsii,

Schmidtii, ,

striatum,

tricolor,

Veischaffeltii, Hort

vesta,

Colocasia, Schott.

antiquorum, Sch., India and Tropies, euit.

Dieffenbachia, Schott.

Bausei, Veitch, Brazil.

Pearcei, Veitch.,

Weiri, Lind et Andre, Brazil.

Homalomena, Sch.

Monstera, Sch.

singraporense, Regel,, Singapore.

deliciosa, Leibm., Mexico.

Philodendron, $\mathrm{Sch}$.

lacerum, Sch., Columbia.

Lindenii, Sch., In

Pothos, $\boldsymbol{L}$.

argyrea, Hort., Johore, Perak.

scandens, $L$, India, Malaya.

Proteinophallus, Hook fil.

campanulatus, Blume., Tropical Asia.

Rivierii, Hk. fll, Cochin China.

Richardia, Knth.

sethiopica, Knth., C, G. Hope.

albo-maculats, Hook, C. G. Hope.

Scindapsus, Schott.

decursivus, Schott., E. Indies, Singapore.

Typhonium, Sehoth.

Pistia, $L$.

Browni, Sehoth., Sub-tropical, Australia.

Stratiotes, L. Malaya.

Aspidistra, Gasel.

ORd, Aspidistase.

punotste, Chine. 
Plectogyne, Baker.

variegata, Baker., Japan

LUACEA, D. C.

Yucea, $L$.

aloifolia, L., Jamaica, \&c.

brevifolia.

glaucescens.

Lilium, $L$. longiflorum, Thueb, Chins.

Washiugtonianum, Kellog. Californis.

Eunkia, Spreng. coerulea, Andr., Japan.

Polianthee, L., Tuberose. tuberose, $I_{f,}, \mathbf{E}$. Indies.

Agapanthus, L'Horit.

umbellatus, L'Herit., C. G. Hope.

Blendfordia, Smith.

Cunninghamis, Lindl., E. Australia.

flammea, Hook, E. Australia,

nobilis, $S m$., Australia.

Phorminm, L., New Zealwil Flag. tenax, Forst., New Zealand.

Sranseviexa, Thunb.

gruineensis, Willd., Guines.

Aloe, $L$. geylaniea, Willd., Ceylon.

Scills, $L$.

carinata, Mill., C. G. Hope.

peruviana, $L_{i n}$, S. Africa.

var, alba.

Ornithogalum, $L$.

caudatum, Ait., C. G. Hope.

Tulbacbia, $L$.

alliacea. $L$, S. Africa

Ord. Melaxthacen, $B$ r.

Methonica, Juss

superba, fam., E. Indies.

Os1). Sambera, Br.

Smilax, Willd.

Sarsaparilla, L., S. America. Sarsaparilla.

syphilitica, $H$. et $B$, Braxil. 
Orb. Asparagze, Le Maont and Dene.

Disnella, Lam. ccerulea, $\mathrm{Rr}$. $\mathrm{Br}$, S. Australia. ensifolia, Redoute., Singapore:!

Cordyline, Commers.

albicans.

ensifolis, Planeh.

Asparagus, $L_{v}$, Asparagus.

ofticinalis, $K_{\text {t., }}$ Europe.

Asparagopsis, $K n t h$.

javaniea, Kuth., Java.

Dracrent, $L$.

Baptistii, Hort, S. S. Islands.

Cooperii, Hort., S. S. Islands.

elliptica, Thanb., Singapore.

Draco, $I_{\text {.., Canaries. }}$

ferrea, L., E. Indies.

var terminalis.

, versicolor.

Fraserii, Hort.

gracilis, Hort.

Guilfoylli.

heliconisefolia, Baker.. Mauritius.

Knoxit, Hort.

metallica, Hort.

marginata, Hort.

nigro-striata, Horl.

sureulosa.

salicifolia, Hort.

Verschaffeltii, Hort.

Ord. X внотірек, Endl.

Xanthorihsea, Smi, Grass trae.

hastilis, F. Muell., 8. Australia.

Ond. Ponfronriacra, A. Rich.

Monochoria, Prsl.

vaginalis, $P_{\text {rsl., Singapore. }}$

Ord. Comanzynaces, Lindl.

Commelyna, Dill.

benghalensis, L., Java and Singapore.

Tradescantin, $L$.

diecolor, L'Herit, S. Americe.

fol. varigata.

zebrina, Hort., S. Ameries. 
Dichorizandra, Mikan.

undata Lindl., Amazon.

Ord. Crpratees, D. C., Sedqes.

Papyrus, Walld.

antiquoram, Willd, Egypt, Puper plant.

Cyperus, $L$.

alternifolius, L, Mndayrascar.

fol. var.

polystachyus, Rttb., Java, Singapore.

tuberosus, Rttb., Jave, Singapore,

Kyllingia, $R u l$.

monocephala, Rttb., Java \& Singapore.

Ord. Graminzen, Jess, Gressrs.

Andropogou, Willd.

Schenanthus, L., Europe, Asia, \&ce, Lemon piuss.

Imperata, Cyriél.

Kuenigii, Rutb, Malaya, Lalang.

Saccharum, L., Sagar Can.

officinarium, $L$., variat. plur. disting.

Anamite bamboo.

" blanche du Cap.

". chinense, due.

". Coke.

". Diard.

". rose.

n)

n

$n$

djong-djoeng.

guingant.

liling.

Large Ribbon.

Malabar.

meerah.

Large Yellow.

ontamite.

mnerai.

reppo.

as

$3 y$

is

it

Oplismenus, Palis.

, var.

Salangore.

soerat.

troboe.

voi=elephant. Cochin-China.

imbecilis, Hort.

Zea, Willd., Maize.

Mays, L., S. America. 
Coix, L., Job's Tears.

Lachryma, $\boldsymbol{L}$, E. Indies.

Reana. Teosinté.

luxurians. Guatemala.

Panicum, $L$.

conjugatum, Thw.? Singapore, inte.

distachyum, $L$., Singapore.

plicatum, Lam., Singapore.

Arundo, $L$.

repens, $L$., Singapore.

Donax, L., S. Europe.

fol. var.

Bambusa, Schreb., Bambon Cane.

giganten, Wall., E. Indies.

nana, Roxb., China.

spinosa, Rxb., Singapore.

stricta, Ruxb., E. Indies.

verticillata, Willd., China.

Beesha, Rheede.

Rheedii, Bedd., Travancore.

travancorensis, Bedd., Travancore.

\section{ACROGENS,}

Ord. Frucres, Ferns.

Gleichenia, $S m$.

[world.

dichotoma, Willd, Trop, and Sub-tropies, new and old flabellata, $B r$., N. Australia to New Zealand.

flagellaris, Spr., Malaye, Mascarene Isles.

Cyathea, $\mathrm{Sm}$. rupestris, Br., N. W. Australia.

dealbata, Sio., New Zealand.

Hookerii, Thw., Ceylon.

sinuata, Hk. \& Gr, Ceylon.

Alsophila, $\mathrm{Br}$.

australis, $\mathrm{Br}$., Australia.

comosa, Hook., Singapore.

excelsa, $\mathrm{Br}$, Norfolk Island.

Cooperii, Hk., New South Wales.

latebrosa, $H k$., Malay Isles.

podophylla, Hk., Hongkong.

tomentosa, $H k$, Formosa.

Youngii, Australia, $=$ Dicksonia ?

Dicksonia, L'Herit.

antaretica, Labill., E. Australia, V. Diemen's Land.

Barometz, Link., Malay Peninsula, (Cibotium Kaulf.)

glaucs, Smith., Sandwich Islands (Cibotium.)

squarrosa, Sw., N. Zealand. 
Hymenophyllum, $L$.

Davallia, Smith. polyanthos, Swarty., Tropies.

affinis, Hook., Tropical Asia.

divaricata, Bheme., Malay Peninsula.

elegaus, Swourtz., Tropies old World.

heterophylli, Swith., Malay Peninsula.

hirta, Kaulf., Malay Peninsula.

mauritiana, $H_{k}$, Mauritius.

parvula, Wallich., Singaporo.

pedata, Smith., Malay Peninsuls.

pentaphylla, Blume, Johore, Perak.

spelunese, Boker., Tropici of Old World.

strigosa, Stoartz,, Malay Pcninsula.

tenuifolia, Swartu., Tropics aud Sub-Tropics Olil World.

vestita, Blume, Singapore.

Lindsaya, Dryand.

ensifolia, Swart., Hongkong.

heterophylla, Dry., Malay Peninsula.

media, $R$. Br., Trop. Australia.

retuse, Melten., Phillipines.

trapeziformis, Dry., Malay Peninsula.

Adiantum, L., Maidenhair Fern.

capillus-Veneris, $L$, Eur. Afr. F. India, S. America.

caudatum, L., Tropics of Old World.

concinnum, Humb, \& Bonp., Trop. Amerien.

letum.

cuncatum, Lang et Frsch., Brazil.

flabellulatum, L., Malay Peninsula, \&e.

formosum, $R$. $B r$., Australin, New Zealand.

hispidulum, Swe. Tropies and Sub.Tropics Old World.

lunulatum, Burm., Tropies everywhere.

macrophyllum, Sw., S. America.

tenerum, Sw., Trop. America.

tetraphyllum, Willd, S. Ameriea.

trapeziforme, $L$., Trop. America.

var. Catherina.

Cheilanthes, siv.

tenufolia, Sw., Trop. and Sub-Trop. old world.

Pellas, Link.

falenta, Fee, Milay Peninsula, \&ce.

geraniafolia, Fee, Trop. Old and New World.

rotundifolia, $\mathrm{Hk}_{k}$, New Zealand and Norfolk Island.

Pteris, L., Brake Wern.

aquilina, $L$., All round the world.

biaurite, L., Tropics Old and New World.

, nemorale, $W_{i} l l d$., Hongkong.

cretica, $L$., Tropics Old and New World. 
albo-lineata, Hort., Brazil.

ensiformis, Burm., Himnlayas to Australia.

incisa, Thunb., Tropical and Sub-Trop. regions.

longifolia, $L$., Trop, and warm resions.

quadriaurits, Retz, All round the world, warm regions.

". asperula, smith.

semipinnata, $L$., India, China, Malays, de.

serrulata, Richard, China, Japan, Natal, \&e. tripartits, Hook., Ceylon, Malaya.

Ceratopteris, Brong.

Lomatia, Willd.

thaliotroides, Brong., Tropics, in quiet waters.

discolor, Willd., Australia and New Zealand.

Blechume, $L$. glauca, Blume., Javs.

orientale, $L$., Himalayas to Australia.

Doodia, R. Br.

Woodwardia, Sw.

aspera, $R$. Br., Temperate Anstralia.

Asplenimm, $L$. Harlandii, Hk., Hongkong.

bantnmense, Baker., E. Indies, \&c., = fraxinifolinm, Wall

Belangerii, Kwaze., Maliy Peninsulia 2,000 ft.

cuneatum, Lom., Hongkong.

lanceum Thumb., Himalayas to Malaya and Chisa.

Nidus, L., Himalayas to Manritius and Howe's Island.

rutefolium, Knnse., Himalnya to Piji=prolongatum, $H_{k}$.

trifurcatum, Hort., Buitewaory=Relangerii.

Thwaitesii, $A . B r$, Ceylon.

viviparum, Presl., Mauritius.

Zcylanicum, Hock, Ceylon.

Scolopendrium, Sin.

rhizoplyyllum, HR., British Amer, to S. U. States.

Aspiditum, Sints.

aristatum, Sne., Himalaysas and Japan to N, S. Wales.

Championii, Benth., Hongkong.

falcatum, Sic, Japan, China, Sandwich Isless.

molle, Sw. Tropics everywhere.

podophyllum. Hoot, Hongkong.

trifoliatum, Sw., Hoin kong, Malay.

undnlatum, Thw., Ceyion.

Nephrodium, Rich,

molle Deso, Warm and Tropical regions.

Thwaitesii (Sagenia), Baker., Ceylon,

Nephrolepis, Schott.

acuta, Presl, Tropics an $\mathrm{S}$ Sub-Tropies = biserrata Schott.

exaltata, Schott.

Duffii, Moore., New Caledonia?" 
Olesndra, Cav,

Cummingii, J. Sm., Malaya. Gunong Gerai. nerïformis, Cav, Trop, and Sub-Tropies.

Polypodium, $L$.

acrostichoides, Forat., Coylon, Malaya to Queensland. conjugatum, Lam., Sub-trop. India, Hongkong, \&c. cucculatum, Nees., Ceylon and Malaya, \&c. difforme, Blume., Bukit Timah, Singapore. Gaminerii, Thw., Ceylon. gramulosum, Presl., Ceylon, Malaya. hemionitideum, Wall, N. India, S. China. irioides, Lam, N. India to Guinea Coast and N. S. Wales. Lingua, $L_{v}, \mathrm{~N}$. India to Malay Islands. nigrescens, Blume., N. India to Friendly Islands. normale, Don., N, India, Malaya, Caffraria. pennigerum, Forst., New Zcaland. Phymatodes, L., Tropics and Sub-Tropics Old World. pteropus, B! unere, N. India to Malaya \& Formoss. quercifolium, $L$, , N. India to Queensland. splendens, Hk: Phillipines and Singapore. tenericanle, Wall., N. India, Malnya \& Chian.

\$ Dipteris, Reinw. bifureatus, Baker., Gunong Pulai, Johore. Horsfieldii, $\boldsymbol{R}$. Br., Malaya to $\mathrm{Fiji}$.

Nothoclæns, $\mathrm{Br}$. suleata, Link., Hongkong.

Gymnogramme, Dest. caudiformis, Mergui to N. Caledonia. javanica. Blume., N. Indit to Sandwich Isls.

Braines, $H h$, Mnlleri, $H k$., Queensland.

Meniscium, sclireb. insignis, Hook., Hongkong and Khasia.

salicifolium, Wall, Penang. simplex, Hk,, Chusan to Malaya. Thwaitesii, IHk, Ceylon. triphyllum, Sw., Himalayas to Malaya.

Antrophyum, Kaulf. 1eticulatum, Kaulf, IHimalayas to Queensiand.

Vittarin, Sw. Scolopendrina, Tho., New Guinea Malay and Mascarene Tienitis, Swe. bleclinoides, Swz, Malaceas and Phillipines.

Drymoglossum, Presl. piloselloides, Presl, Himalayas to Malaya.

Acrostichum, $L$. appendieulatum, Willd., Himalnyas to Malayas aud aureum, $L$., Tropiss and Sub-Tropics. auritum, Swo, Malaya. 


\section{4}

decurrens, Hook., =variable $H \hbar$., Mulaya, \&c.

latifolium, Sw., S. America.

quercifolium, Rets., India, Ceylon, Cochin China.

repandum, Blume., Phillipines and Malaya, \&c.

rigidum, Wall., Malaya.

sorbifolium, $L_{n}$, Tropies and Sub-Tropics.

viscosum, Sac., Tropics and Sub-Tropies.

Platycerium, Desv.

biforme, $B l$., Singapore.

grande J. Siw., Singapore.

Hillii, Moore, Queensland.

Osmunda, $L$.

jaranica Blome., Kamschatka to Java and Ceylon.

Schizza, Sisith.

dichotomn, . Sw., Tropics and Sub-Tropics.

malaccana, Baker., Perak.

Lygodium, Stw.

dichotomum, Sw., Chusan to Malaya.

japonicum, Sw.. Japan to Malaya.

standens, Stw S. China to Malaya.

Angiopteris, Họjim.

evecta Hoffm., Himalayas to Society Isles.

Marattia, Sw.

" hypoleuca, Jasa.

fraxises, Swith., Tropies and Sub-Tropies of old world.

Ophioglossum, $L$.

pendulum, L., Singapore, Ceylon, \&ce., \&c.

Helminthostachys, Kaulf.

zeylanica, $H h$., Himalayas to Quceusland.

\section{LYCORODTACEA.}

Lycopodium, $\boldsymbol{L}$.

ecrnuum, L., E. Indies Malaya Chinn.

flabellulatam, $L_{\text {., }}$ Chima Malaya.

laxum, $B l$., Malaya.

Psilotium, Sw.

Phlegmaria, L., Malay Península, Java.

flaccidum, Wall., Johore.

Selaginella, $P$. de $B$.

cesia, $L$., Malaya, Singapore.

var, arborea.

cuspidata.

denticulatum.

erythropus, spreng., Trop. America.

Griflithii.

insequalifoliz, Hk, \& Gr., E. Indie, Malaya.

Kruaseanum.

Wallichii. Johore, Perak. 


\section{PALMS OMITTED TROM PALMACE}

Rhopaloblasta.

Calyptronoma.

hexandra, Scheffer. Java.

Swartzi. Jamaics.

Borassus, L.

flabelliformis, India und Malaya, Longtar.

Latania, Comm.

borbonice, Lam. Malagascar.

glaucophylla, Lodd. Mauritius.

rubra, Jace. Mauritins:

Lodoicea, Labill. Double Cocoenuat.

seychellarum, Labill. Seychelles.

Hyphane, Grin. Doum Paln. thebaica, Mart. Egypt.

Bentinekia, Berry.

Coddapanna, B. Indies.

Coryphs, $L$.

australis, R. Pr., Australia.

clata, Rosb. Malay Peninsula.

Haliera, Roub. E. Indies.

umbraculifera, $L$. F. Indies, Talipot.

Iivistona, R. Br.

altissima, Zoll, Java.

humilis, $R$. Br. Australia.

olivaeformis, Mart. Java.

rotundifolia, Mart. Jara, Atalay Peninsula.

Pritchardia, Beem.

fllamentoss, Lind. Arizona.

pacifica, Seem. S. S. Islands.

1st Jomuary, 1879.

H. J. MURTON, Superintendent, gc.

Total spp. and varieties.

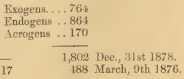

1,802 Dec., 31 st 1878.

188 March, 9 th 7876.

Plants in old Catalogue dead $\overline{17}$

Increase, 1,314

H. J. MUURTON, 29- $9-79$ 
Conserved by

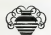

National Archives of Singapore 
